\title{
Estimación de la esperanza de vida a nivel municipal y por marginación sociodemográfica: una aplicación del método de Swanson para el caso de México, 2010
}

\author{
Estimation of life expectancy at the municipal level \\ and sociodemographic marginalization: Using \\ the Swanson method for the case of Mexico, 2010
}

Israel Paredes*

Eliud Silva**

\begin{abstract}
Resumen
Se presenta la aplicación del método de Swanson, modelo de regresión no lineal, para estimar la esperanza de vida al nacer para el caso de México en 2010, tanto a nivel municipal como por grado de marginación sociodemográfica. Se evidencia la simplicidad del método a través de exponer los pocos insumos demográficos que requiere, y se concluye con el potencial y precisión de la herramienta por grado de marginación, así como con la coherencia que se alcanza al hacer comparaciones entre las estimaciones obtenidas en la aplicación y los datos que se tienen de manera oficial del indicador.
\end{abstract}

Palabras clave: esperanza de vida; marginación sociodemográfica; regresión no lineal; mortalidad.

\begin{abstract}
The Swanson method, a nonlinear regression model, is used to estimate life expectancy at birth for the case of Mexico 2010, at both the municipal level and because of the sociodemographic degree of marginalization. The authors show the simplicity of the method by explaining the few demographic inputs required, and concludes with the potential and accuracy of the tool due to the degree of marginalization, as well as the consistency achieved when making comparisons between the estimates obtained in the application and the data obtained from the official indicator.
\end{abstract}

Key words: life expectancy; sociodemographic marginalization; nonlinear regression; mortality.

* Universidad Anáhuac México, Facultad de Ciencias Actuariales. Dirección postal: Av. Universidad Anáhuac 46, 52786, Huixquilucan, Estado de México, México. Correo electrónico: <israelp11@hotmail.com>.

**- Universidad Anáhuac México, Facultad de Ciencias Actuariales. Dirección postal: Av. Universidad Anáhuac 46, 52786, Huixquilucan, Estado de México, México. Correo electrónico: <jsilvaurrutia@hotmail.com>.

ESTUDIOS DEMOGRÁFICOS Y URBANOS, VOL. 32, NÚM. 1 (94), 2017, pp. 97-129 


\section{Introducción}

La esperanza de vida se define como el número de años de vida que restan, término medio, a una persona de no variar la tendencia de la mortalidad (Haupt y Kane, 2003: 58). En otras palabras, es una síntesis de las tasas específicas de mortalidad. El indicador resume la intensidad de la mortalidad que experimenta una población, ya sea al nacimiento o en una edad alcanzada específica. Con frecuencia la esperanza de vida se utiliza como indicador de desarrollo humano o bienestar, como en el Programa de las Naciones Unidas para el Desarrollo (PNUD, 2012); también se suele emplear para fines comparativos entre poblaciones residentes en distintas unidades geográficas (países, entidades, zonas metropolitanas, etc.). Resulta importante su monitoreo de manera periódica con el fin de estudiar su evolución respecto a sí misma, garantizar la comparabilidad con otras poblaciones, y en ocasiones, propiciar la toma de decisiones informada en materia de salud pública y desarrollo social. En México, el Consejo Nacional de Población (Conapo) es quien calcula oficialmente la esperanza de vida.

El cálculo de dicho indicador puede resultar laborioso y más a nivel municipal, pues aun con el avance de los equipos y los programas de cómputo más potentes, implica un alto costo en términos del manejo estadístico de información, tanto por el volumen como por el empleo de datos censales y registros administrativos de defunciones. Para 2010 se tienen, según datos del Instituto Nacional de Estadística y Geografía (INEGI), un total de 2454 municipios; con ello, siguiendo la metodología clásica para la estimación de la esperanza de vida, $\mathrm{e}_{\mathrm{x}}$, se tendrían que calcular 2454 tablas de mortalidad. Generalmente, para el caso mexicano, se calcula el indicador en cada ejercicio censal y se enfoca, sobre todo, a poblaciones grandes, es decir a las entidades o al país en su totalidad. Esto hace que la disponibilidad del indicador para unidades geográficas pequeñas, como lo pueden ser algunos municipios o localidades, sea escasa o inexistente.

Por otra parte, en ocasiones las políticas públicas al ser valoradas por su impacto, en términos del número de familias, hogares o personas beneficiadas, ponen en desventaja a aquellas unidades geográficas que implican un bajo impacto, es decir unidades con poblaciones pequeñas. Por esto, dejar de lado el cálculo de la esperanza de vida para unidades geográficas con baja cantidad de población podría ocultar sus posibles necesidades apremiantes. Es por ello que resulta deseable tener un mecanismo que permita hacer estimaciones de la esperanza de vida, de manera precisa, con un bajo costo en cuanto al manejo de información estadístico-demográfica y con una notable celeridad en relación con lo que se obtiene con el método 
clásico de estimación del indicador. También es un hecho que contar con el parámetro de esperanza de vida a nivel estatal propicia que se "escondan" las particularidades y diversidad (o bien, la desigualdad) inherentes a las entidades. Por ejemplo, las condiciones de vida en el municipio de Acapulco, valoradas a través del índice de marginación sociodemográfico de 2010 (Conapo, 2011), son notablemente distintas a las que se tienen en otros municipios del estado de Guerrero. Mientras que en Acapulco la marginación es considerada Baja, en el 75\% de los municipios de Guerrero se considera Alta o Muy alta.

De acuerdo con el INEGI (2001), para 1930 la esperanza de vida al nacer para mujeres era de 35 años, en tanto que para hombres era de 33. Para el 2010, la esperanza de vida se situaba en 77 y 71 años respectivamente. En 2014, los estados con mayor valor en el indicador, es decir con población en promedio más longeva, son Nuevo León y el Distrito Federal, con 76 años, seguidos por Baja California Sur con 75.9 años. Por otra parte, el estado con menor esperanza de vida oficial es Chihuahua, con 72.3 años; es de llamar la atención este caso, pues se trata de una entidad con bajo grado de marginación sociodemográfico (Conapo, 2015). Sería oportuno realizar un estudio para explicar las causas que lo generan.

Dado lo estratégico e informativo que resulta el indicador de esperanza de vida al nacer, y que de manera oficial no está disponible a nivel municipal, en este trabajo se busca exponer el método propuesto por Swanson (1989) e ilustrar su uso y simplicidad para estimarlo para una gran cantidad de municipios de México. Asimismo se agregan las estimaciones por grado de marginación con la finalidad de contrastar la precisión del método con los datos oficiales. A partir de dichas estimaciones se valora la heterogeneidad del indicador en el interior de las entidades del país. Cabe advertir que este trabajo no busca cuestionar el modelo, ni proponer variantes o alternativas en su estimación. Se considera que hacerlo representa una rica e interesante línea de investigación ajena al objetivo de este trabajo.

Las estadísticas vitales y los datos de las poblaciones utilizados fueron extraídos de organismos oficiales como la Secretaría de Salud (SS), el INEGI y el Conapo. Aun cuando es cuestionable que exista la misma calidad de la información para todas las unidades geográficas del país, por priorizar la exposición del manejo del método y su aplicación, se asume que ésta en general es aceptable y similar en todo México. Se considera que un mecanismo útil para mitigar y estandarizar las eventuales discrepancias en la calidad de la información es el método de Guerrero y Silva (2013), en el que se estiman tendencias y se impone una suavidad para hacer comparativos, $o$ bien el de Silva y Guerrero (2016), donde se elaboran estimaciones de ten- 
dencias en superficies de mortalidad en las dimensiones de edad y años con imposición de suavidades para efectos comparativos entre países nórdicos.

El documento está organizado de la siguiente manera. En la próxima sección se citan algunos antecedentes en cuanto a metodologías para calcular la esperanza de vida en general y para áreas pequeñas. En la sección siguiente se señala la metodología empleada para elaborar las estimaciones a nivel municipal y se aborda la definición del índice de marginación propuesto por el Conapo. Posteriormente se documentan los resultados generados, proporcionándolos a través de cuadros y un mapa. Finalmente se presentan las conclusiones y los comentarios derivados de los hallazgos obtenidos de la elaboración del presente trabajo.

\section{Marco teórico}

La estimación clásica de la esperanza de vida se elabora grosso modo de la siguiente manera. Por un lado, se computan las defunciones registradas en las respectivas estadísticas vitales, que en México administra la SS en coordinación con el INEGI. Por otro lado, se obtiene el total de la población por sexo, edad y espacio geográfico. Finalmente se estiman las tasas de mortalidad al realizar los respectivos cocientes del primero respecto al segundo, y se multiplica por algún múltiplo de 100 o 1000. Más adelante se realiza la estimación del resto de las columnas de la tabla de mortalidad, hasta llegar a la estimación de la última columna, que se refiere a la esperanza de vida a la edad x, e (véase Chiang, 1968; Ortega, 1982 y 1987).

Para el cálculo de la esperanza de vida a nivel municipal, el enfoque del método clásico de estimación puede resultar, además de costoso, impreciso. De acuerdo con Swanson (1989), y con las referencias que ahí se citan, se tienen cuatro grandes perspectivas para calcular la esperanza de vida: 1) la basada en el principio de poblaciones estables; 2) la justificada en teorías biológicas de envejecimiento; 3) la basada en la estimación de poblaciones por edad y 4) la sustentada en ecuaciones de regresión donde se vincula la esperanza de vida con otros indicadores demográficos. Es justamente en este último bloque donde se ubica la propuesta que se aplica en este documento.

Eayres y Williams (2003) comparan distintos modelos de estimación de la esperanza de vida. Entre otros hallazgos, señalan que los modelos propuestos por Chiang (1968) y Silcocks et al. (2001) generan estimaciones puntuales y errores estándar similares. Asimismo, los autores citan como hallazgo que la población debe de ser de al menos 5000 habitantes para ofrecer estimaciones confiables. Por otro lado, Swanson y Tedrow (2012) 
muestran cómo el uso de modelos de supervivencia censales puede ser útil para estimar la esperanza de vida cuando la población a estudiar no experimenta una alta migración, en tanto que Sarma y Choudhury (2014) estudian diferentes escenarios en una población con información faltante y concluyen que el mejor modelo para estimar la esperanza de vida es el que se compone de un modelo de regresión. Un amplio marco de referencia para estimar la esperanza de vida a través de modelos de regresión en áreas pequeñas se encuentra en Congdon (2014a).

Existen diversas aplicaciones de la propuesta utilizada en este trabajo, entre las cuales destacan: a) en Swanson et al. (2009) se realiza un estudio de las diferencias en las ganancias en la esperanza de vida para comunidades norteamericanas en estratos económicos opuestos de 1970 a 1990, encontrándose menos favorecidas aquellas comunidades con bajo estrato socioeconómico; y b) en Flores et al. (2013) se estima la esperanza de vida en municipios mexicanos, haciendo agregaciones por tamaño de localidad en términos de población y ubicación geográfica, y se miden las diferencias entre 1990 y 2000. Se concluye que las comunidades pequeñas son las menos favorecidas en ganancia de esperanza de vida por el desarrollo económico. Cabe mencionar que en Flores et al. (2013) se propone una agregación geográfica y poblacional específica, distinta a la realizada en este trabajo. En el presente documento se hace uso del índice de marginación y se propone la "fusión" de municipios con escasa información, para tener así mayor cobertura poblacional. Dado que se adoptaron distintas agregaciones, no es posible hacer comparaciones entre los resultados alcanzados en ambos trabajos.

En lo tocante a la estimación de esperanza de vida para áreas pequeñas, Toson y Baker (2005) hacen un recorrido entre las distintas opciones que existen en la literatura, elaboran comparativos y enfatizan lo relativo a tamaños mínimos de población, así como en el caso de ausencia de muertes en determinada edad. Por otro lado, Bravo y Malta (2010) emplean técnicas de graduación paramétricas para suavizar tasas específicas de mortalidad, para con ello estimar modelos de supervivencia. Asimismo, en Congdon (2014b) se ejemplifican estimaciones ante cambios en la esperanza de vida en Estados Unidos. Aun cuando se considera altamente recomendable el uso de estas últimas propuestas para el caso mexicano, se propone en este trabajo tener una aproximación de las esperanzas de vida exclusivamente a través del método de Swanson (1989), incluyendo el uso del índice de marginación sociodemográfica, con propósitos de agregación, para así reflejar objetivamente las características de los municipios. 


\section{Índice de marginación sociodemográfica}

Un elemento sustantivo que se usa en este trabajo es el índice de marginación sociodemográfica que estima el Conapo desde 1990. Éste se elabora con el objetivo de dimensionar las posibles carencias que sufre la población en cada entidad, municipio o localidad del país (Conapo, 2011). El índice se estima mediante el método estadístico de componentes principales, con lo que se sintetiza la información de nueve indicadores provenientes del Censo de Población y Vivienda o del Conteo correspondiente. Después de calcular el valor del índice, se clasifica a cada entidad, municipio o localidad en uno de cinco grupos (grados de marginación).

Las variables consideradas para estimar el índice se engloban en los siguientes bloques:

1) Educación (porcentaje de población de 15 años o más analfabeta y porcentaje de población de 15 años o más sin primaria completa).

2) Vivienda (porcentaje de ocupantes en viviendas sin drenaje ni excusado, porcentaje de ocupantes en viviendas sin energía eléctrica, porcentaje de ocupantes en viviendas sin agua entubada, porcentaje de viviendas con algún nivel de hacinamiento, y porcentaje de ocupantes en viviendas con piso de tierra).

3) Distribución de la población (porcentaje de población en localidades con menos de 5000 habitantes).

4) Ingresos monetarios (porcentaje de población ocupada con ingresos de hasta dos salarios mínimos).

Refiriéndose al grado de marginación por entidad, se siguió el resultado obtenido por el Conapo (2011), que a nivel de entidades federativas se resume como sigue:

- Muy alto: Guerrero, Chiapas, Oaxaca.

- Alto: Veracruz, Puebla, Hidalgo, San Luis Potosí, Michoacán, Tabasco, Campeche, Yucatán.

- Medio: Nayarit, Zacatecas, Guanajuato, Durango, Tlaxcala, Sinaloa, Querétaro, Morelos, Quintana Roo.

- Bajo: Chihuahua, Estado de México, Baja California Sur, Sonora, Tamaulipas, Colima, Jalisco, Aguascalientes.

- Muy bajo: Coahuila, Baja California, Nuevo León, Distrito Federal. 


\section{Metodología}

Se utiliza el modelo de regresión pionero propuesto por Mazur (1972) y refinado por Swanson (1989). Utilizar este modelo de regresión no lineal, según este último autor, tiene las siguientes ventajas:

- No se asume estabilidad o cuasi-estabilidad en la población.

- Los datos necesarios para el cálculo son generalmente de fácil acceso y sólo son tres: $a$ ) total de muertes, $b$ ) población total y c) población de 65 y más años.

- Es preciso para el caso en que se tengan grandes poblaciones.

- A pesar de que su uso se ha extendido a áreas pequeñas de manera menos extensiva que a áreas grandes, los resultados han demostrado tener una alta precisión.

- Es posible realizar estimaciones en momentos intercensales de manera oportuna.

El modelo se compone de dos variables: la tasa bruta de mortalidad (CDR por sus siglas en inglés), definida como el total de defunciones entre el total de población (se utiliza población a mitad de año), y el porcentaje de la población con 65 y más años ( $\mathrm{P} 65+$ ); ambas observadas en un mismo año calendario. El modelo fue resultado de la experimentación y culminó en la siguiente expresión:

$$
\begin{aligned}
e_{0}=\beta_{0}+\beta_{1} C D R+ & \beta_{2} \ln (P 65+)+\beta_{3} C D R^{2}+\beta_{4} \ln (P 65+)^{2} \\
& +\beta_{5} C D R \ln (P 65+)
\end{aligned}
$$

donde

$e_{0}$ : es la esperanza de vida al nacer

$\beta_{0}, \beta_{1}, \beta_{2}, \beta_{3}, \beta_{4}, \beta_{5}$ : son parámetros a estimar.

El modelo funciona de manera más eficiente cuando se construye la esperanza de vida al nacer para poblaciones que no se consideren (áreas) pequeñas. Como señala el autor, el modelo no es apropiado si: $a$ ) la población es "especial", por ejemplo si está envejecida con más de $20 \%$ de población de 65 y más años; y b) la comunidad es pequeña y con pocas muertes, en específico 50 o menos, tal que la CDR fluctúe de manera considerable año 
con año. Para aplicar el modelo, es recomendable hacer un suavizamiento en las muertes, haciendo un promedio con los años anterior y posterior respecto al año a estudiar, es decir información trianual. Los supuestos de los errores en el marco de los modelos de regresión no lineal son: media cero, independencia, homocedasticidad, normalidad y estar idénticamente distribuidos (Davidian y Giltinan, 1995).

Para las estimaciones respectivas que se ilustran, así como para el mapa que se presenta más adelante, se utilizan los softwares estadísticos SPSS y R. Los datos necesarios para estimar el modelo para los municipios en México en 2010 fueron obtenidos de la SS y del INEGI. Se desea ser enfático en que se asume que la calidad de los datos es suficiente para la realización de las estimaciones. Luego se calcularon las variables CDR y P65+, y se identificaron aquellos municipios que no cumplían con los requisitos necesarios para la estimación. Con ello, los municipios que quedan excluidos se agregan entre sí de acuerdo con el estado al que pertenecen y a su grado de marginación; esto con el propósito de abarcar a la mayor cantidad de población dentro de la estimación. Se puede afirmar que ésta se hace en dos etapas: primero a nivel estatal y luego en el municipal.

Posteriormente se seleccionan los municipios de acuerdo con su grado de marginación (Muy alto, Alto, Medio, Bajo y Muy bajo) y se aplica el modelo para cada uno de estos grados, teniendo como variable dependiente la esperanza de vida oficial que el Conapo asigna al estado donde se ubica el municipio. Más adelante se realizan pruebas estadísticas de significancia de parámetros estimados y se elabora el análisis de residuales. Con las estimaciones para cada municipio se hace un promedio ponderado de la esperanza de vida de acuerdo con el grado de marginación en los estados y se compara contra un promedio ponderado de la esperanza de vida oficial por grado de marginación.

Se identifica que Chihuahua representa un caso especial e inusual en relación con el resto, pues es un estado con baja marginación sociodemográfica, pero con la esperanza de vida más baja. Al proceder de manera similar, en este caso no se obtenían resultados con sentido demográfico en relación con el dato oficial, por lo que se utilizó otra estrategia. Después de varios ensayos, se decidió no realizar suavizamiento entre tres años de referencia y se agrupó de acuerdo con lo sugerido por Flores (2013), es decir, se conjuntó a Chihuahua con los estados descritos como "Sur" (Campeche, Chiapas, Guerrero, Oaxaca, Quintana Roo, Tabasco, Veracruz y Yucatán). Posteriormente se estimó la regresión y se validaron los supuestos estadísticos de significancia y los residuales. Es importante enfatizar que si no se tenía un tratamiento especial en este caso, se sobreestimaba 
en cinco años la esperanza de vida de la entidad y en consecuencia se afectaba la capacidad predictiva del modelo en general. El intentar plantear hipótesis o explicar las causas de dicha coyuntura escapa al objetivo de este documento.

\section{Resultados}

Para verificar el poder predictivo de la aplicación del modelo de regresión se realizó una prueba preliminar para calcular la esperanza de vida al nacer, utilizando las cifras a nivel estatal. Se debe tener en cuenta que se trata de una aproximación inicial y que ésta se afina más adelante. El cuadro 1 muestra la esperanza de vida al nacer que ofrece el Conapo, así como las diferencias en relación con las estimaciones realizadas. Con base en los resultados se puede apreciar cómo en algunas entidades la aproximación es bastante precisa, como lo es en el caso de Querétaro. Las máximas discrepancias se presentan, siendo mayores las estimaciones que los datos oficiales, en los estados de Chiapas, Guerrero y Oaxaca. En sentido opuesto ocurre en el caso de Nuevo León. Cabe notar que el promedio de las diferencias fue apenas de -0.0003125 .

En el cuadro 2 se muestra el resumen de las estimaciones del modelo y sus respectivos estadísticos (entre paréntesis los valores del estadístico $t$ de Student). Se valora que todos los parámetros estimados son estadísticamente significativos, que existe una relación funcional entre la variable dependiente y las independientes a través del valor del estadístico F, y que la varianza explicada de la esperanza de vida al nacer es del orden del $64 \%$. Asimismo se verifica el cumplimiento de los supuestos del modelo.

Una vez verificadas las estimaciones iniciales, se procede con la estimación de la esperanza de vida a nivel municipal. Bajo las condiciones en las cuales el modelo es apropiado según lo señalado por Swanson (1989), es necesario excluir algunos municipios. En el cuadro 3 se muestra el total de municipios por grado de marginación sociodemográfica, el número de municipios seleccionados, aquellos no seleccionados, así como otros porcentajes.

El 52\% del total de los municipios existentes según el Censo de Población y Vivienda de 2010 quedan seleccionados y puede apreciarse la siguiente relación: a menor grado de marginación, mayor cantidad de municipios incluidos en las estimaciones. Esto sugiere que el método, como se señaló anteriormente, es más eficiente a mayor tamaño de los municipios en términos poblacionales. Ahora, en el cuadro 4 se presenta el porcentaje de la población que queda contenida dentro de los municipios seleccionados por grado de marginación. Debe notarse que los totales de este cuadro se incrementan 


\section{Cuadro 1}

Datos oficiales y estimaciones de la esperanza de vida, 2010

\begin{tabular}{|c|c|c|c|}
\hline Entidad & Oficial & Estimado & Diferencia \\
\hline Aguascalientes & 75.19 & 74.67 & 0.52 \\
\hline Baja California & 73.07 & 72.88 & 0.19 \\
\hline Baja California Sur & 75.50 & 75.10 & 0.40 \\
\hline Campeche & 74.64 & 74.20 & 0.44 \\
\hline Coahuila & 74.71 & 74.36 & 0.35 \\
\hline Colima & 75.25 & 74.36 & 0.89 \\
\hline Chiapas & 71.95 & 74.76 & -2.81 \\
\hline Chihuahua & 68.68 & 68.95 & -0.27 \\
\hline Distrito Federal & 75.49 & 75.11 & 0.38 \\
\hline Durango & 73.92 & 74.60 & -0.68 \\
\hline Guanajuato & 74.66 & 74.20 & 0.46 \\
\hline Guerrero & 71.91 & 73.20 & -1.29 \\
\hline Hidalgo & 73.62 & 73.39 & 0.23 \\
\hline Jalisco & 74.76 & 74.54 & 0.22 \\
\hline México & 74.45 & 74.85 & -0.40 \\
\hline Michoacán & 73.84 & 73.74 & 0.10 \\
\hline Morelos & 74.72 & 74.10 & 0.62 \\
\hline Nayarit & 73.86 & 73.67 & 0.19 \\
\hline Nuevo León & 75.79 & 74.47 & 1.32 \\
\hline Oaxaca & 71.93 & 73.24 & -1.31 \\
\hline Puebla & 74.00 & 74.51 & -0.51 \\
\hline Querétaro & 74.68 & 74.69 & -0.01 \\
\hline Quintana Roo & 74.95 & 74.73 & 0.22 \\
\hline San Luis Potosí & 73.76 & 72.88 & 0.88 \\
\hline Sinaloa & 73.84 & 74.44 & -0.60 \\
\hline Sonora & 74.52 & 74.33 & 0.19 \\
\hline Tabasco & 73.99 & 74.26 & -0.27 \\
\hline Tamaulipas & 74.72 & 74.48 & 0.24 \\
\hline Tlaxcala & 74.34 & 73.90 & 0.44 \\
\hline Veracruz & 73.29 & 74.64 & -1.35 \\
\hline Yucatán & 74.90 & 74.69 & 0.21 \\
\hline Zacatecas & 74.31 & 73.31 & 1.00 \\
\hline
\end{tabular}

Fuente: Para los datos oficiales, Conapo, 2012; para los datos estimados, elaboración propia con base en INEGI, 2010a y $2010 \mathrm{~b}$. 
Paredes y Silva, ESTIMACIÓN DE LA ESPERANZA DE VIDA

\section{Cuadro 2}

Estimación de los coeficientes del modelo nivel estatal, 2010

\begin{tabular}{lc}
\hline Variable & Coeficiente de regresión \\
\hline cte & -405.7 \\
$(\mathrm{t})$ & -5.03 \\
$\mathrm{CDR}$ & 52.18 \\
$(\mathrm{t})$ & 5.94 \\
$\mathrm{CDR}{ }^{\wedge} 2$ & -1.145 \\
$(\mathrm{t})$ & -4.17 \\
$\ln (\mathrm{P} 65+)$ & -245.22 \\
$(\mathrm{t})$ & -5.77 \\
$\ln (\mathrm{P} 65+)^{\wedge} 2$ & -30.44 \\
$(\mathrm{t})$ & -5.4 \\
$\mathrm{CDR} * \ln (\mathrm{P} 65+)$ & 14.29 \\
$(\mathrm{t})$ & 6.33 \\
& \\
$\mathrm{~F}$ & 9.56 \\
$\mathrm{R}^{2}$ & 0.64 \\
\hline
\end{tabular}

Fuente: Elaboración propia con base en INEGI, 2010a y 2010b.

debido a que, como estrategia de estimación, algunos municipios se aglomeran en municipios ficticios, es decir se fusionaron bloques de unidades geográficas donde se considera el total de sus defunciones y/o población mayor a 65 años. De esta manera se mejora la cobertura de las estimaciones.

Una vez estimadas las esperanzas de vida para cada municipio se inicia una reconstrucción de la esperanza de vida por grado de marginación como medida de consistencia de las estimaciones. Así, se toma el valor obtenido de $\mathrm{e}_{\mathrm{x}}$ del modelo de regresión y se calcula un promedio ponderado con la población total de cada municipio de acuerdo con su grado de marginación. Ésta se compara con un promedio ponderado de los datos oficiales del Conapo a nivel estatal, agrupando de igual manera por grado de marginación. El cuadro 5 muestra el estimado promedio y las diferencias.

En el mapa 1 se muestra la esperanza de vida en México a nivel municipal para 2010. Para esto se utilizaron los respectivos archivos geográficos del INEGI. Debe notarse que las unidades geográficas que están en blanco no se incluyeron en la estimación. Salvo el caso de Chihuahua, se podría apreciar grosso modo un patrón de mejores niveles de esperanza de vida al 


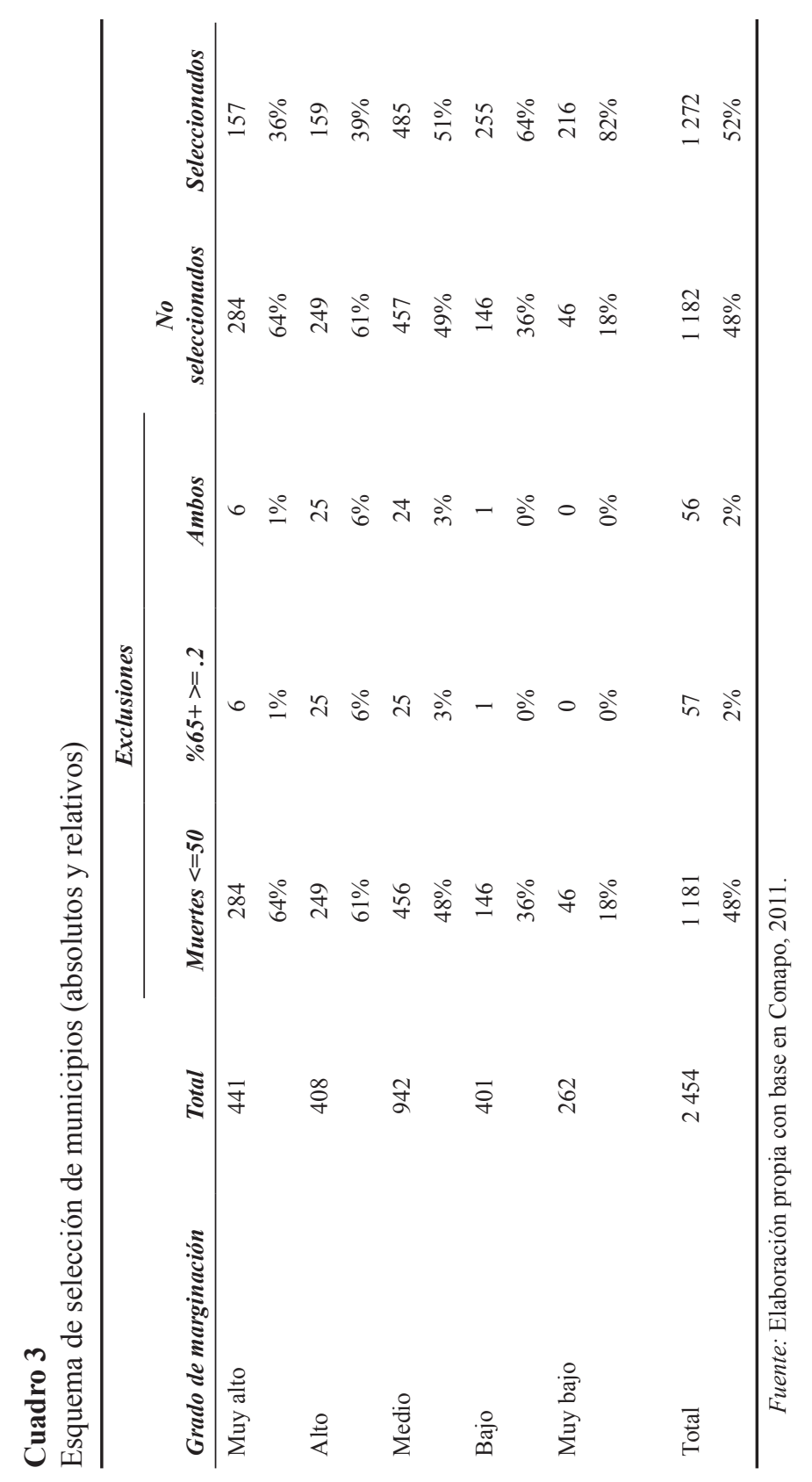




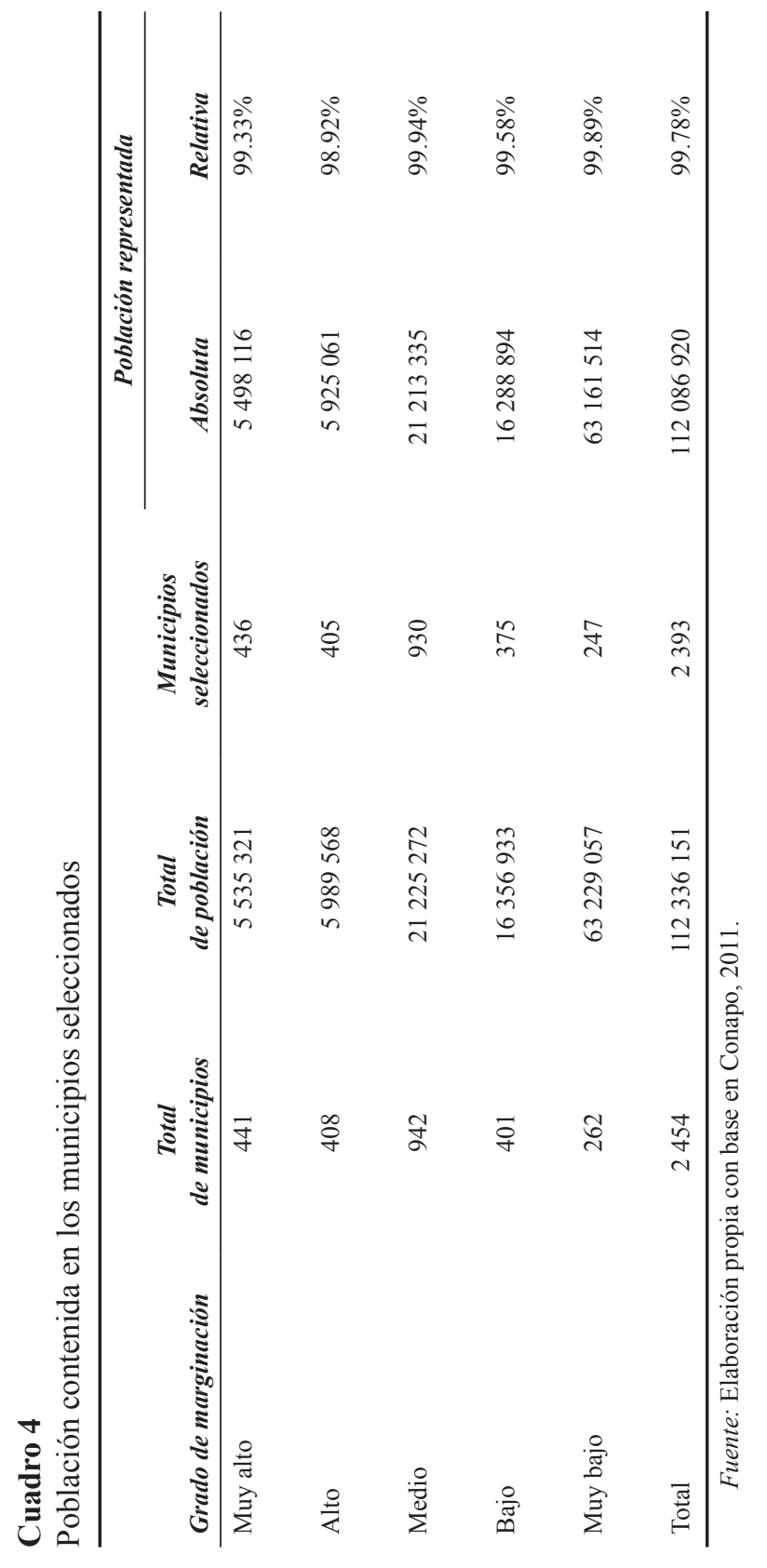




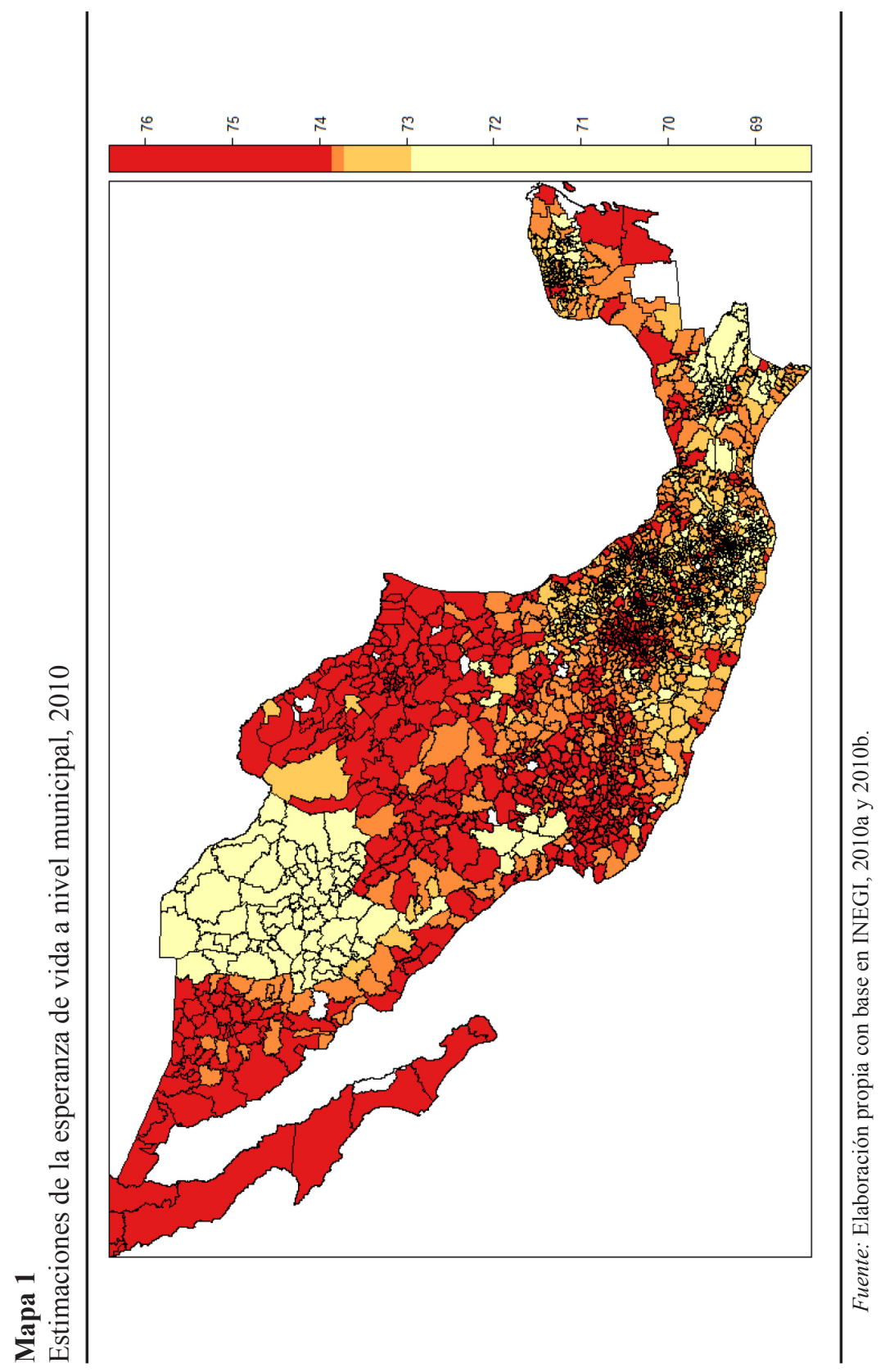




\section{Cuadro 5}

Estimado de la esperanza de vida, 2010

\begin{tabular}{lccc}
\hline Grado de marginación & Oficial & Estimado & Diferencia \\
\hline Muy alto & 72.57 & 72.71 & -0.14 \\
Alto & 73.13 & 73.13 & 0.00 \\
Medio & 73.79 & 73.81 & -0.02 \\
Bajo & 74.03 & 74.19 & -0.17 \\
Muy bajo & 74.56 & 74.47 & 0.09 \\
\hline
\end{tabular}

Fuente: Para los datos oficiales, elaboración propia con base en Conapo, 2012; para los datos estimados, con base en INEGI, 2010a y 2010 b.

norte del país, así como en zonas con alta densidad demográfica. En el anexo se muestra el cuadro con las esperanzas de vida municipales estimadas. Debe tenerse presente que el hecho de aspirar a una cobertura mayor de municipios implica que aproximadamente la mitad de los resultados son representativos para el bloque de municipios con características similares.

Finalmente en el cuadro 6 se ilustran las desviaciones estándar de las estimaciones de las esperanzas de vida al nacer a nivel de entidad federativa; esto como una medida de heterogeneidad del indicador dentro de las entidades. Algo relevante digno de destacar es que no necesariamente el grado de marginación estatal implica la existencia de mayor heterogeneidad en las correspondientes esperanzas de vida de la población de los municipios. Por ejemplo, véanse los casos de Quintana Roo, Chiapas, Nayarit y Nuevo León, por un lado. Y por otro los casos de Baja California, Tabasco, Zacatecas, Aguascalientes y Distrito Federal.

\section{Conclusiones}

Se considera que queda de manifiesto la viabilidad de estimar esperanzas de vida a nivel municipal por medio del modelo propuesto por Swanson para el caso mexicano, haciendo uso de la agregación por marginación sociodemográfica, tanto en el caso de los municipios que cumplen con los supuestos exigidos, como por bloques de municipios. Se logra obtener estimados de la esperanza de vida al nacer para una gran cantidad de municipios del país de manera sucinta. Las condiciones del modelo restringen la inclusión de comunidades pequeñas que con frecuencia son marginadas, pero su aglomeración es un elemento que ayuda a corregir en alguna medida su omisión. Sin embargo, para los casos en los que se desee una estimación 
ESTUDIOS DEMOGRÁFICOS Y URBANOS, VOL. 32, NÚM. 1 (94), 2017, pp. 97-129

\section{Cuadro 6}

Variaciones de la esperanza de vida por entidad, 2010

\begin{tabular}{|c|c|}
\hline Entidad & Desviación estándar \\
\hline Aguascalientes & 0.246 \\
\hline Baja California & 0.011 \\
\hline Baja California Sur & 0.354 \\
\hline Campeche & 0.354 \\
\hline Coahuila & 0.293 \\
\hline Colima & 0.406 \\
\hline Chiapas & 0.646 \\
\hline Chihuahua & 0.285 \\
\hline Distrito Federal & 0.283 \\
\hline Durango & 0.424 \\
\hline Guanajuato & 0.295 \\
\hline Guerrero & 0.421 \\
\hline Hidalgo & 0.438 \\
\hline Jalisco & 0.399 \\
\hline México & 0.472 \\
\hline Michoacán & 0.352 \\
\hline Morelos & 0.307 \\
\hline Nayarit & 0.608 \\
\hline Nuevo León & 0.568 \\
\hline Oaxaca & 0.426 \\
\hline Puebla & 0.374 \\
\hline Querétaro & 0.359 \\
\hline Quintana Roo & 0.867 \\
\hline San Luis Potosí & 0.419 \\
\hline Sinaloa & 0.379 \\
\hline Sonora & 0.489 \\
\hline Tabasco & 0.184 \\
\hline Tamaulipas & 0.454 \\
\hline Tlaxcala & 0.319 \\
\hline Veracruz & 0.492 \\
\hline Yucatán & 0.404 \\
\hline Zacatecas & 0.186 \\
\hline
\end{tabular}

Fuente: Elaboración propia con base en INEGI, 2010a y $2010 \mathrm{~b}$. 
individual del municipio, sería apropiado aplicar otras metodologías como las referentes a áreas pequeñas.

Las estimaciones proporcionadas por el modelo, siendo agrupadas por grado de marginación, muestran diferencias muy pequeñas en relación con los datos oficiales. De hecho, se aprecia tanto en las estimaciones como en los datos oficiales que a mayor grado de marginación, menor es la esperanza de vida (a excepción de Chihuahua). Esto contribuye a evidenciar que las condiciones sociodemográficas desfavorables repercuten directamente en la esperanza de vida de la población, haciendo notable la necesidad de atención de una política pública en estos sectores.

El caso de Chihuahua merece atención especial, pues la asignación de la esperanza de vida estatal a los municipios incluidos en el modelo de regresión altera de manera significativa los estimadores de los parámetros del conjunto, resultando en una peor estimación para los demás municipios. Se podría pensar que la baja esperanza de vida corresponde a un grado de marginación elevado, pero no es así; el índice de marginación para el estado es de -0.53 , que corresponde a un grado bajo de marginación. Por esta razón, es tomado el estado como un dato atípico y excluido en una primera instancia del modelo, para su posterior incorporación, como fue citado en la metodología. No obstante, se deja abierta la posibilidad de un futuro estudio donde se profundice en explicar las causas que provocan dicho comportamiento.

Una aportación que se considera relevante en este trabajo es la de aplicar la metodología referida para el caso mexicano y su sencilla implementación comparada con metodologías tradicionales. Ahora queda claro que no hace falta tener exclusivamente datos en años censales para hacer la estimación de la esperanza de vida a nivel municipal; cada año, con proyecciones del Conapo y con estadísticas vitales de defunciones, es factible desarrollar tales cálculos. Sería interesante extender el modelo para poder asociar intervalos de estimación con determinado nivel y/o supuestos de distribución en las estimaciones puntuales que proporciona la aplicación del modelo. 
ESTUDIOS DEMOGRÁFICOS Y URBANOS, VOL. 32, NÚM. 1 (94), 2017, pp. 97-129

\section{Anexo}

\section{Cuadro A1}

Esperanzas de vida estimadas a nivel municipal, 2010

\begin{tabular}{|c|c|c|c|c|c|c|c|c|}
\hline Ent. & Mun. & $\boldsymbol{e}_{0}$ & Ent. & Mun. & $e_{0}$ & Ent. & Mun. & $e_{0}$ \\
\hline 1 & 1 & 74.3 & 5 & 18 & 74.3 & 7 & 14 & 72.0 \\
\hline 1 & 2 & 73.8 & 5 & 20 & 74.7 & 7 & 15 & 73.8 \\
\hline 1 & 3 & 74.2 & 5 & 22 & 74.4 & 7 & 16 & 73.8 \\
\hline 1 & 5 & 74.5 & 5 & 24 & 74.1 & 7 & 17 & 73.8 \\
\hline 1 & 6 & 74.2 & 5 & 25 & 74.3 & 7 & 19 & 73.8 \\
\hline 1 & 7 & 74.2 & 5 & 27 & 74.4 & 7 & 20 & 72.9 \\
\hline 1 & 11 & 74.4 & 5 & 28 & 74.8 & 7 & 21 & 73.8 \\
\hline 2 & 1 & 74.3 & 5 & 30 & 74.3 & 7 & 22 & 71.4 \\
\hline 2 & 2 & 74.3 & 5 & 31 & 74.8 & 7 & 23 & 72.5 \\
\hline 2 & 3 & 74.4 & 5 & 32 & 74.6 & 7 & 26 & 72.0 \\
\hline 2 & 4 & 74.3 & 5 & 33 & 74.2 & 7 & 27 & 73.8 \\
\hline 2 & 5 & 74.3 & 5 & 35 & 74.3 & 7 & 30 & 72.8 \\
\hline 3 & 1 & 74.2 & 5 & 36 & 73.8 & 7 & 31 & 71.9 \\
\hline 3 & 2 & 74.2 & 5 & 38 & 74.2 & 7 & 32 & 73.2 \\
\hline 3 & 3 & 74.3 & 6 & 1 & 73.8 & 7 & 34 & 73.9 \\
\hline 3 & 8 & 74.9 & 6 & 2 & 74.5 & 7 & 35 & 73.2 \\
\hline 4 & 1 & 73.8 & 6 & 3 & 74.2 & 7 & 37 & 73.2 \\
\hline 4 & 2 & 74.4 & 6 & 4 & 74.2 & 7 & 38 & 72.8 \\
\hline 4 & 3 & 74.2 & 6 & 5 & 75.2 & 7 & 39 & 72.4 \\
\hline 4 & 4 & 73.8 & 6 & 7 & 74.3 & 7 & 40 & 73.8 \\
\hline 4 & 5 & 73.8 & 6 & 9 & 74.1 & 7 & 41 & 73.0 \\
\hline 4 & 6 & 73.8 & 6 & 10 & 74.4 & 7 & 44 & 72.8 \\
\hline 4 & 9 & 73.9 & 7 & 1 & 73.8 & 7 & 46 & 73.8 \\
\hline 4 & 11 & 73.1 & 7 & 2 & 73.8 & 7 & 47 & 72.3 \\
\hline 5 & 2 & 74.4 & 7 & 3 & 73.3 & 7 & 48 & 73.8 \\
\hline 5 & 3 & 74.6 & 7 & 4 & 72.0 & 7 & 49 & 71.8 \\
\hline 5 & 4 & 74.2 & 7 & 5 & 72.6 & 7 & 51 & 73.8 \\
\hline 5 & 6 & 74.7 & 7 & 6 & 73.1 & 7 & 52 & 72.4 \\
\hline 5 & 7 & 74.2 & 7 & 8 & 72.8 & 7 & 54 & 73.3 \\
\hline 5 & 9 & 74.2 & 7 & 9 & 73.8 & 7 & 57 & 73.0 \\
\hline 5 & 10 & 74.5 & 7 & 11 & 73.1 & 7 & 59 & 71.6 \\
\hline 5 & 11 & 73.9 & 7 & 12 & 72.7 & 7 & 60 & 72.7 \\
\hline 5 & 17 & 74.2 & 7 & 13 & 72.2 & 7 & 61 & 72.9 \\
\hline
\end{tabular}


Paredes y Silva, ESTIMACIÓN DE LA ESPERANZA DE VIDA

\section{Cuadro A1}

\section{(continúa)}

\begin{tabular}{|c|c|c|c|c|c|c|c|c|}
\hline Ent. & Mun. & $e_{0}$ & Ent. & Mun. & $e_{0}$ & Ent. & Mun. & $e_{0}$ \\
\hline 7 & 62 & 72.8 & 7 & 109 & 72.6 & 8 & 62 & 68.7 \\
\hline 7 & 64 & 72.5 & 7 & 110 & 72.7 & 8 & 63 & 68.6 \\
\hline 7 & 65 & 72.5 & 7 & 112 & 71.9 & 8 & 65 & 68.5 \\
\hline 7 & 66 & 72.0 & 7 & 113 & 71.6 & 9 & 2 & 74.7 \\
\hline 7 & 68 & 73.8 & 7 & 115 & 71.9 & 9 & 3 & 75.2 \\
\hline 7 & 69 & 73.3 & 8 & 1 & 68.4 & 9 & 4 & 74.4 \\
\hline 7 & 70 & 72.9 & 8 & 2 & 69.1 & 9 & 5 & 74.8 \\
\hline 7 & 71 & 73.2 & 8 & 3 & 69.0 & 9 & 6 & 75.1 \\
\hline 7 & 72 & 72.2 & 8 & 5 & 68.8 & 9 & 7 & 74.4 \\
\hline 7 & 74 & 74.3 & 8 & 7 & 68.4 & 9 & 8 & 74.7 \\
\hline 7 & 75 & 72.9 & 8 & 8 & 69.8 & 9 & 9 & 74.2 \\
\hline 7 & 76 & 72.4 & 8 & 9 & 68.4 & 9 & 10 & 74.6 \\
\hline 7 & 77 & 72.6 & 8 & 10 & 68.7 & 9 & 11 & 74.5 \\
\hline 7 & 78 & 74.0 & 8 & 11 & 68.9 & 9 & 12 & 74.4 \\
\hline 7 & 79 & 73.9 & 8 & 12 & 68.8 & 9 & 13 & 74.6 \\
\hline 7 & 80 & 72.6 & 8 & 17 & 69.0 & 9 & 14 & 74.8 \\
\hline 7 & 81 & 71.9 & 8 & 19 & 69.0 & 9 & 15 & 74.3 \\
\hline 7 & 83 & 73.1 & 8 & 21 & 68.9 & 9 & 16 & 74.7 \\
\hline 7 & 86 & 73.8 & 8 & 25 & 68.7 & 9 & 17 & 75.1 \\
\hline 7 & 87 & 72.9 & 8 & 27 & 68.5 & 10 & 1 & 74.2 \\
\hline 7 & 89 & 73.8 & 8 & 28 & 69.1 & 10 & 4 & 74.2 \\
\hline 7 & 92 & 73.1 & 8 & 29 & 69.0 & 10 & 5 & 74.3 \\
\hline 7 & 93 & 72.2 & 8 & 31 & 68.6 & 10 & 7 & 74.3 \\
\hline 7 & 94 & 72.6 & 8 & 32 & 68.7 & 10 & 8 & 74.2 \\
\hline 7 & 97 & 73.8 & 8 & 35 & 68.9 & 10 & 9 & 73.8 \\
\hline 7 & 98 & 72.0 & 8 & 36 & 68.7 & 10 & 12 & 74.5 \\
\hline 7 & 100 & 72.5 & 8 & 37 & 68.5 & 10 & 13 & 73.8 \\
\hline 7 & 101 & 74.5 & 8 & 40 & 68.8 & 10 & 14 & 72.6 \\
\hline 7 & 102 & 72.2 & 8 & 45 & 68.6 & 10 & 16 & 73.9 \\
\hline 7 & 103 & 73.1 & 8 & 48 & 69.0 & 10 & 17 & 73.9 \\
\hline 7 & 104 & 73.1 & 8 & 50 & 68.8 & 10 & 18 & 74.3 \\
\hline 7 & 105 & 73.8 & 8 & 52 & 68.8 & 10 & 20 & 73.9 \\
\hline 7 & 106 & 73.2 & 8 & 53 & 69.0 & 10 & 22 & 74.2 \\
\hline 7 & 107 & 73.1 & 8 & 54 & 69.2 & 10 & 23 & 73.9 \\
\hline 7 & 108 & 73.8 & 8 & 55 & 68.6 & 10 & 24 & 73.9 \\
\hline
\end{tabular}


ESTUDIOS DEMOGRÁFICOS Y URBANOS, VOL. 32, NÚM. 1 (94), 2017, pp. 97-129

\section{Cuadro A1}

\section{(continúa)}

\begin{tabular}{|c|c|c|c|c|c|c|c|c|}
\hline Ent. & Mun. & $e_{0}$ & Ent. & Mun. & $e_{0}$ & Ent. & Mun. & $e_{0}$ \\
\hline 10 & 26 & 73.8 & 11 & 29 & 73.8 & 12 & 26 & 72.8 \\
\hline 10 & 28 & 73.9 & 11 & 30 & 73.8 & 12 & 27 & 72.9 \\
\hline 10 & 32 & 73.8 & 11 & 31 & 74.2 & 12 & 28 & 72.9 \\
\hline 10 & 34 & 72.8 & 11 & 32 & 73.9 & 12 & 29 & 74.2 \\
\hline 10 & 35 & 73.9 & 11 & 33 & 73.8 & 12 & 30 & 73.3 \\
\hline 10 & 36 & 74.2 & 11 & 35 & 73.8 & 12 & 32 & 72.9 \\
\hline 10 & 38 & 74.2 & 11 & 37 & 73.9 & 12 & 33 & 73.8 \\
\hline 10 & 39 & 74.2 & 11 & 38 & 73.9 & 12 & 34 & 73.2 \\
\hline 11 & 1 & 73.8 & 11 & 39 & 73.9 & 12 & 35 & 74.1 \\
\hline 11 & 2 & 74.2 & 11 & 40 & 73.2 & 12 & 38 & 74.2 \\
\hline 11 & 3 & 73.8 & 11 & 41 & 74.1 & 12 & 39 & 73.8 \\
\hline 11 & 4 & 73.8 & 11 & 42 & 73.8 & 12 & 40 & 73.2 \\
\hline 11 & 5 & 73.8 & 11 & 43 & 73.8 & 12 & 41 & 73.0 \\
\hline 11 & 7 & 74.3 & 11 & 44 & 74.2 & 12 & 42 & 73.0 \\
\hline 11 & 8 & 73.8 & 11 & 46 & 73.8 & 12 & 44 & 73.8 \\
\hline 11 & 9 & 73.8 & 12 & 1 & 74.1 & 12 & 45 & 73.0 \\
\hline 11 & 10 & 73.8 & 12 & 2 & 73.0 & 12 & 46 & 72.9 \\
\hline 11 & 11 & 74.2 & 12 & 3 & 73.0 & 12 & 48 & 73.8 \\
\hline 11 & 12 & 73.8 & 12 & 4 & 73.0 & 12 & 49 & 73.8 \\
\hline 11 & 13 & 73.8 & 12 & 6 & 73.3 & 12 & 50 & 73.8 \\
\hline 11 & 14 & 73.8 & 12 & 7 & 73.8 & 12 & 51 & 73.0 \\
\hline 11 & 15 & 74.4 & 12 & 10 & 72.8 & 12 & 52 & 72.9 \\
\hline 11 & 16 & 73.8 & 12 & 11 & 73.8 & 12 & 53 & 73.0 \\
\hline 11 & 17 & 74.4 & 12 & 12 & 72.7 & 12 & 54 & 73.0 \\
\hline 11 & 18 & 74.2 & 12 & 13 & 73.0 & 12 & 55 & 73.8 \\
\hline 11 & 19 & 73.8 & 12 & 14 & 73.9 & 12 & 56 & 73.0 \\
\hline 11 & 20 & 74.3 & 12 & 15 & 73.9 & 12 & 57 & 73.8 \\
\hline 11 & 21 & 75.1 & 12 & 16 & 73.0 & 12 & 58 & 73.3 \\
\hline 11 & 22 & 73.8 & 12 & 17 & 73.2 & 12 & 59 & 73.2 \\
\hline 11 & 23 & 73.8 & 12 & 18 & 73.4 & 12 & 61 & 73.8 \\
\hline 11 & 24 & 73.9 & 12 & 19 & 73.0 & 12 & 62 & 72.8 \\
\hline 11 & 25 & 74.3 & 12 & 20 & 73.0 & 12 & 63 & 72.9 \\
\hline 11 & 26 & 73.8 & 12 & 21 & 73.3 & 12 & 64 & 73.0 \\
\hline 11 & 27 & 74.5 & 12 & 22 & 73.0 & 12 & 66 & 72.9 \\
\hline 11 & 28 & 73.9 & 12 & 23 & 73.3 & 12 & 67 & 73.3 \\
\hline
\end{tabular}


Paredes y Silva, ESTIMACIÓN DE LA ESPERANZA DE VIDA

\section{Cuadro A1}

\section{(continúa)}

\begin{tabular}{|c|c|c|c|c|c|c|c|c|}
\hline Ent. & Mun. & $e_{0}$ & Ent. & Mun. & $e_{0}$ & Ent. & Mun. & $e_{0}$ \\
\hline 12 & 68 & 73.3 & 13 & 35 & 73.8 & 13 & 84 & 73.8 \\
\hline 12 & 71 & 72.8 & 13 & 37 & 73.3 & 14 & 1 & 73.8 \\
\hline 12 & 72 & 72.9 & 13 & 40 & 72.9 & 14 & 2 & 74.6 \\
\hline 12 & 73 & 73.0 & 13 & 41 & 74.2 & 14 & 3 & 75.1 \\
\hline 12 & 74 & 73.0 & 13 & 44 & 73.9 & 14 & 5 & 74.2 \\
\hline 12 & 75 & 73.8 & 13 & 46 & 73.4 & 14 & 6 & 74.2 \\
\hline 12 & 76 & 72.3 & 13 & 48 & 74.2 & 14 & 8 & 74.2 \\
\hline 12 & 77 & 73.8 & 13 & 49 & 73.4 & 14 & 9 & 74.6 \\
\hline 12 & 79 & 72.7 & 13 & 50 & 74.2 & 14 & 13 & 74.2 \\
\hline 12 & 81 & 73.0 & 13 & 51 & 74.5 & 14 & 15 & 74.5 \\
\hline 13 & 1 & 73.8 & 13 & 52 & 74.2 & 14 & 16 & 73.8 \\
\hline 13 & 2 & 72.9 & 13 & 53 & 73.0 & 14 & 17 & 73.9 \\
\hline 13 & 3 & 74.1 & 13 & 54 & 73.8 & 14 & 18 & 74.1 \\
\hline 13 & 5 & 74.2 & 13 & 55 & 73.8 & 14 & 21 & 74.2 \\
\hline 13 & 6 & 73.9 & 13 & 56 & 74.2 & 14 & 22 & 74.2 \\
\hline 13 & 8 & 74.1 & 13 & 57 & 73.8 & 14 & 23 & 74.3 \\
\hline 13 & 10 & 74.5 & 13 & 58 & 73.8 & 14 & 24 & 74.2 \\
\hline 13 & 11 & 73.4 & 13 & 59 & 73.8 & 14 & 25 & 74.2 \\
\hline 13 & 12 & 73.8 & 13 & 60 & 73.8 & 14 & 27 & 73.4 \\
\hline 13 & 13 & 74.2 & 13 & 61 & 74.7 & 14 & 29 & 73.8 \\
\hline 13 & 14 & 73.3 & 13 & 62 & 73.0 & 14 & 30 & 75.2 \\
\hline 13 & 15 & 73.8 & 13 & 63 & 74.2 & 14 & 33 & 73.8 \\
\hline 13 & 16 & 73.8 & 13 & 67 & 73.8 & 14 & 35 & 74.2 \\
\hline 13 & 17 & 73.9 & 13 & 69 & 74.5 & 14 & 36 & 75.1 \\
\hline 13 & 18 & 73.4 & 13 & 71 & 73.3 & 14 & 37 & 75.2 \\
\hline 13 & 19 & 73.8 & 13 & 73 & 73.2 & 14 & 39 & 74.4 \\
\hline 13 & 23 & 74.2 & 13 & 74 & 74.2 & 14 & 42 & 73.9 \\
\hline 13 & 24 & 73.8 & 13 & 75 & 74.2 & 14 & 43 & 73.8 \\
\hline 13 & 25 & 73.2 & 13 & 76 & 74.4 & 14 & 44 & 74.3 \\
\hline 13 & 27 & 73.0 & 13 & 77 & 74.1 & 14 & 45 & 73.8 \\
\hline 13 & 28 & 73.8 & 13 & 78 & 73.0 & 14 & 46 & 74.2 \\
\hline 13 & 29 & 73.8 & 13 & 80 & 73.0 & 14 & 47 & 74.2 \\
\hline 13 & 30 & 73.8 & 13 & 81 & 73.8 & 14 & 48 & 73.8 \\
\hline 13 & 31 & 73.9 & 13 & 82 & 74.2 & 14 & 50 & 74.2 \\
\hline 13 & 34 & 72.8 & 13 & 83 & 74.2 & 14 & 53 & 74.2 \\
\hline
\end{tabular}


ESTUDIOS DEMOGRÁFICOS Y URBANOS, VOL. 32, NÚM. 1 (94), 2017, pp. 97-129

\section{Cuadro A1}

\section{(continúa)}

\begin{tabular}{|c|c|c|c|c|c|c|c|c|}
\hline Ent. & Mun. & $e_{0}$ & Ent. & Mun. & $e_{0}$ & Ent. & Mun. & $e_{0}$ \\
\hline 14 & 55 & 74.1 & 14 & 110 & 74.2 & 15 & 30 & 74.4 \\
\hline 14 & 58 & 74.2 & 14 & 113 & 73.8 & 15 & 31 & 74.5 \\
\hline 14 & 59 & 74.2 & 14 & 114 & 74.2 & 15 & 32 & 72.8 \\
\hline 14 & 61 & 72.9 & 14 & 116 & 74.2 & 15 & 33 & 74.3 \\
\hline 14 & 63 & 74.4 & 14 & 118 & 74.3 & 15 & 35 & 74.9 \\
\hline 14 & 64 & 73.8 & 14 & 119 & 74.2 & 15 & 36 & 74.2 \\
\hline 14 & 65 & 73.9 & 14 & 120 & 74.4 & 15 & 37 & 74.5 \\
\hline 14 & 66 & 74.2 & 14 & 121 & 75.1 & 15 & 39 & 74.6 \\
\hline 14 & 67 & 74.3 & 14 & 123 & 73.8 & 15 & 40 & 73.8 \\
\hline 14 & 68 & 73.9 & 14 & 124 & 74.2 & 15 & 42 & 73.8 \\
\hline 14 & 69 & 74.0 & 14 & 125 & 74.2 & 15 & 43 & 73.9 \\
\hline 14 & 70 & 74.5 & 15 & 1 & 73.8 & 15 & 44 & 74.4 \\
\hline 14 & 73 & 74.2 & 15 & 2 & 74.4 & 15 & 45 & 73.8 \\
\hline 14 & 74 & 74.2 & 15 & 3 & 73.8 & 15 & 47 & 73.8 \\
\hline 14 & 77 & 74.2 & 15 & 4 & 73.8 & 15 & 48 & 73.8 \\
\hline 14 & 78 & 74.2 & 15 & 5 & 74.0 & 15 & 50 & 74.2 \\
\hline 14 & 82 & 74.7 & 15 & 7 & 73.8 & 15 & 51 & 74.4 \\
\hline 14 & 83 & 74.4 & 15 & 8 & 73.3 & 15 & 52 & 73.8 \\
\hline 14 & 84 & 73.8 & 15 & 9 & 74.1 & 15 & 53 & 74.4 \\
\hline 14 & 85 & 74.2 & 15 & 10 & 74.2 & 15 & 54 & 74.3 \\
\hline 14 & 86 & 73.8 & 15 & 11 & 74.3 & 15 & 56 & 73.8 \\
\hline 14 & 87 & 73.9 & 15 & 13 & 74.5 & 15 & 57 & 74.6 \\
\hline 14 & 88 & 74.2 & 15 & 14 & 73.9 & 15 & 58 & 74.7 \\
\hline 14 & 91 & 74.2 & 15 & 15 & 73.8 & 15 & 59 & 74.4 \\
\hline 14 & 92 & 74.0 & 15 & 16 & 73.8 & 15 & 60 & 74.4 \\
\hline 14 & 93 & 74.4 & 15 & 18 & 74.3 & 15 & 62 & 74.4 \\
\hline 14 & 94 & 74.2 & 15 & 19 & 74.4 & 15 & 63 & 73.8 \\
\hline 14 & 96 & 74.2 & 15 & 20 & 74.3 & 15 & 64 & 73.8 \\
\hline 14 & 97 & 74.7 & 15 & 21 & 73.8 & 15 & 65 & 74.2 \\
\hline 14 & 98 & 74.4 & 15 & 23 & 74.2 & 15 & 67 & 74.0 \\
\hline 14 & 100 & 73.8 & 15 & 24 & 74.5 & 15 & 68 & 74.2 \\
\hline 14 & 101 & 74.5 & 15 & 25 & 74.3 & 15 & 70 & 74.2 \\
\hline 14 & 105 & 74.2 & 15 & 26 & 73.8 & 15 & 71 & 73.8 \\
\hline 14 & 108 & 74.2 & 15 & 28 & 74.2 & 15 & 74 & 72.8 \\
\hline 14 & 109 & 73.8 & 15 & 29 & 74.7 & 15 & 75 & 74.2 \\
\hline
\end{tabular}


Paredes y Silva, ESTIMACIÓN DE LA ESPERANZA DE VIDA

\section{Cuadro A1}

\section{(continúa)}

\begin{tabular}{|c|c|c|c|c|c|c|c|c|}
\hline Ent. & Mun. & $e_{0}$ & Ent. & Mun. & $e_{0}$ & Ent. & Mun. & $e_{0}$ \\
\hline 15 & 76 & 74.4 & 15 & 118 & 74.3 & 16 & 38 & 73.3 \\
\hline 15 & 80 & 73.0 & 15 & 119 & 73.2 & 16 & 40 & 73.8 \\
\hline 15 & 81 & 74.5 & 15 & 120 & 74.4 & 16 & 41 & 73.8 \\
\hline 15 & 82 & 73.8 & 15 & 121 & 74.3 & 16 & 42 & 73.9 \\
\hline 15 & 84 & 74.2 & 15 & 122 & 74.4 & 16 & 43 & 74.2 \\
\hline 15 & 85 & 73.8 & 15 & 123 & 73.4 & 16 & 44 & 74.1 \\
\hline 15 & 86 & 73.8 & 15 & 124 & 72.9 & 16 & 45 & 74.2 \\
\hline 15 & 87 & 74.0 & 16 & 1 & 73.8 & 16 & 47 & 73.8 \\
\hline 15 & 88 & 73.8 & 16 & 2 & 73.8 & 16 & 49 & 73.3 \\
\hline 15 & 90 & 74.2 & 16 & 3 & 73.8 & 16 & 50 & 73.8 \\
\hline 15 & 91 & 74.4 & 16 & 4 & 73.9 & 16 & 51 & 74.2 \\
\hline 15 & 92 & 74.5 & 16 & 5 & 73.8 & 16 & 52 & 74.4 \\
\hline 15 & 93 & 74.2 & 16 & 6 & 73.8 & 16 & 53 & 74.4 \\
\hline 15 & 94 & 74.2 & 16 & 8 & 73.0 & 16 & 54 & 74.0 \\
\hline 15 & 95 & 74.4 & 16 & 9 & 73.8 & 16 & 55 & 73.8 \\
\hline 15 & 96 & 74.5 & 16 & 10 & 73.8 & 16 & 56 & 73.4 \\
\hline 15 & 97 & 73.8 & 16 & 12 & 73.8 & 16 & 57 & 73.0 \\
\hline 15 & 99 & 74.3 & 16 & 15 & 73.8 & 16 & 58 & 74.2 \\
\hline 15 & 100 & 74.4 & 16 & 16 & 74.0 & 16 & 60 & 73.8 \\
\hline 15 & 101 & 74.2 & 16 & 17 & 73.8 & 16 & 61 & 73.8 \\
\hline 15 & 102 & 73.8 & 16 & 19 & 73.8 & 16 & 62 & 73.8 \\
\hline 15 & 103 & 74.6 & 16 & 20 & 73.8 & 16 & 63 & 74.0 \\
\hline 15 & 104 & 74.7 & 16 & 21 & 73.4 & 16 & 64 & 73.8 \\
\hline 15 & 105 & 73.3 & 16 & 22 & 73.8 & 16 & 65 & 73.8 \\
\hline 15 & 106 & 74.2 & 16 & 24 & 73.8 & 16 & 66 & 74.1 \\
\hline 15 & 107 & 74.2 & 16 & 25 & 73.8 & 16 & 67 & 74.0 \\
\hline 15 & 108 & 74.4 & 16 & 29 & 73.0 & 16 & 68 & 74.2 \\
\hline 15 & 109 & 74.5 & 16 & 30 & 74.0 & 16 & 69 & 74.5 \\
\hline 15 & 110 & 74.1 & 16 & 31 & 73.8 & 16 & 70 & 74.2 \\
\hline 15 & 111 & 72.9 & 16 & 32 & 73.9 & 16 & 71 & 73.8 \\
\hline 15 & 112 & 73.8 & 16 & 33 & 73.8 & 16 & 72 & 73.8 \\
\hline 15 & 113 & 73.9 & 16 & 34 & 73.8 & 16 & 73 & 73.8 \\
\hline 15 & 114 & 72.6 & 16 & 35 & 73.3 & 16 & 74 & 73.9 \\
\hline 15 & 115 & 74.2 & 16 & 36 & 74.2 & 16 & 75 & 73.8 \\
\hline 15 & 117 & 73.0 & 16 & 37 & 74.2 & 16 & 76 & 74.1 \\
\hline
\end{tabular}


ESTUDIOS DEMOGRÁFICOS Y URBANOS, VOL. 32, NÚM. 1 (94), 2017, pp. 97-129

\section{Cuadro A1}

\section{(continúa)}

\begin{tabular}{|c|c|c|c|c|c|c|c|c|}
\hline Ent. & Mun. & $e_{0}$ & Ent. & Mun. & $e_{0}$ & Ent. & Mun. & $e_{0}$ \\
\hline 16 & 77 & 73.3 & 17 & 4 & 73.8 & 18 & 15 & 73.8 \\
\hline 16 & 78 & 73.9 & 17 & 6 & 74.4 & 18 & 16 & 73.9 \\
\hline 16 & 79 & 73.8 & 17 & 7 & 74.5 & 18 & 17 & 74.3 \\
\hline 16 & 80 & 73.8 & 17 & 8 & 74.4 & 18 & 18 & 74.2 \\
\hline 16 & 82 & 73.8 & 17 & 9 & 74.2 & 18 & 20 & 74.4 \\
\hline 16 & 83 & 73.8 & 17 & 10 & 73.8 & 19 & 4 & 74.9 \\
\hline 16 & 84 & 73.8 & 17 & 11 & 74.6 & 19 & 5 & 74.2 \\
\hline 16 & 85 & 73.9 & 17 & 12 & 74.1 & 19 & 6 & 75.0 \\
\hline 16 & 86 & 74.2 & 17 & 13 & 73.8 & 19 & 7 & 73.9 \\
\hline 16 & 87 & 74.2 & 17 & 15 & 73.8 & 19 & 9 & 74.6 \\
\hline 16 & 88 & 74.3 & 17 & 16 & 73.8 & 19 & 11 & 75.1 \\
\hline 16 & 89 & 73.8 & 17 & 17 & 73.8 & 19 & 12 & 74.5 \\
\hline 16 & 91 & 73.9 & 17 & 18 & 74.2 & 19 & 13 & 75.2 \\
\hline 16 & 92 & 73.0 & 17 & 19 & 73.8 & 19 & 14 & 73.9 \\
\hline 16 & 93 & 73.8 & 17 & 20 & 74.2 & 19 & 17 & 73.9 \\
\hline 16 & 94 & 74.1 & 17 & 21 & 73.9 & 19 & 18 & 75.6 \\
\hline 16 & 95 & 74.2 & 17 & 22 & 73.8 & 19 & 19 & 75.2 \\
\hline 16 & 97 & 73.0 & 17 & 24 & 74.2 & 19 & 21 & 74.8 \\
\hline 16 & 98 & 73.8 & 17 & 25 & 73.8 & 19 & 22 & 74.3 \\
\hline 16 & 99 & 73.0 & 17 & 28 & 74.2 & 19 & 26 & 74.6 \\
\hline 16 & 100 & 73.8 & 17 & 29 & 74.2 & 19 & 31 & 75.4 \\
\hline 16 & 102 & 74.4 & 17 & 30 & 73.8 & 19 & 33 & 74.9 \\
\hline 16 & 103 & 73.8 & 17 & 31 & 74.6 & 19 & 38 & 74.2 \\
\hline 16 & 104 & 73.9 & 18 & 1 & 73.8 & 19 & 39 & 74.5 \\
\hline 16 & 105 & 74.2 & 18 & 2 & 74.2 & 19 & 44 & 75.1 \\
\hline 16 & 106 & 74.2 & 18 & 3 & 74.0 & 19 & 45 & 74.3 \\
\hline 16 & 107 & 74.2 & 18 & 4 & 74.2 & 19 & 46 & 74.5 \\
\hline 16 & 108 & 74.1 & 18 & 6 & 75.1 & 19 & 48 & 74.4 \\
\hline 16 & 110 & 73.8 & 18 & 7 & 73.8 & 19 & 49 & 75.0 \\
\hline 16 & 111 & 73.8 & 18 & 8 & 74.5 & 20 & 2 & 73.3 \\
\hline 16 & 112 & 73.8 & 18 & 9 & 72.6 & 20 & 5 & 74.0 \\
\hline 16 & 113 & 73.9 & 18 & 10 & 73.8 & 20 & 6 & 73.8 \\
\hline 17 & 1 & 73.8 & 18 & 11 & 73.8 & 20 & 10 & 74.2 \\
\hline 17 & 2 & 74.2 & 18 & 12 & 74.2 & 20 & 14 & 74.2 \\
\hline 17 & 3 & 73.8 & 18 & 14 & 73.8 & 20 & 21 & 73.8 \\
\hline
\end{tabular}


Paredes y Silva, ESTIMACIÓN DE LA ESPERANZA DE VIDA

\section{Cuadro A1}

\section{(continúa)}

\begin{tabular}{|c|c|c|c|c|c|c|c|c|}
\hline Ent. & Mun. & $e_{0}$ & Ent. & Mun. & $e_{0}$ & Ent. & Mun. & $e_{0}$ \\
\hline 20 & 26 & 73.1 & 20 & 325 & 72.9 & 21 & 23 & 73.0 \\
\hline 20 & 28 & 73.3 & 20 & 334 & 73.4 & 21 & 26 & 73.9 \\
\hline 20 & 39 & 74.1 & 20 & 335 & 71.8 & 21 & 34 & 73.9 \\
\hline 20 & 41 & 73.0 & 20 & 385 & 74.3 & 21 & 35 & 73.8 \\
\hline 20 & 43 & 73.8 & 20 & 386 & 72.6 & 21 & 36 & 73.0 \\
\hline 20 & 44 & 73.8 & 20 & 391 & 72.7 & 21 & 41 & 74.5 \\
\hline 20 & 57 & 73.8 & 20 & 397 & 73.7 & 21 & 43 & 73.0 \\
\hline 20 & 58 & 73.0 & 20 & 398 & 72.3 & 21 & 44 & 73.8 \\
\hline 20 & 59 & 73.8 & 20 & 407 & 72.7 & 21 & 45 & 73.8 \\
\hline 20 & 67 & 74.3 & 20 & 414 & 73.2 & 21 & 47 & 73.9 \\
\hline 20 & 68 & 73.8 & 20 & 438 & 72.9 & 21 & 48 & 73.8 \\
\hline 20 & 73 & 73.8 & 20 & 440 & 74.1 & 21 & 49 & 72.9 \\
\hline 20 & 79 & 74.4 & 20 & 448 & 72.4 & 21 & 50 & 72.7 \\
\hline 20 & 85 & 72.7 & 20 & 467 & 73.8 & 21 & 51 & 73.8 \\
\hline 20 & 115 & 73.0 & 20 & 468 & 72.8 & 21 & 53 & 73.8 \\
\hline 20 & 124 & 73.0 & 20 & 469 & 73.0 & 21 & 54 & 73.9 \\
\hline 20 & 134 & 72.7 & 20 & 470 & 72.8 & 21 & 58 & 72.9 \\
\hline 20 & 151 & 73.3 & 20 & 482 & 73.8 & 21 & 61 & 72.7 \\
\hline 20 & 171 & 73.0 & 20 & 483 & 73.7 & 21 & 63 & 73.8 \\
\hline 20 & 177 & 73.9 & 20 & 551 & 73.8 & 21 & 64 & 73.3 \\
\hline 20 & 184 & 74.1 & 20 & 553 & 73.8 & 21 & 65 & 73.8 \\
\hline 20 & 190 & 73.3 & 20 & 559 & 73.8 & 21 & 67 & 73.8 \\
\hline 20 & 198 & 73.0 & 20 & 561 & 72.8 & 21 & 69 & 73.3 \\
\hline 20 & 205 & 73.0 & 20 & 567 & 72.8 & 21 & 71 & 73.8 \\
\hline 20 & 207 & 73.0 & 20 & 569 & 73.3 & 21 & 72 & 73.0 \\
\hline 20 & 232 & 72.9 & 21 & 1 & 73.9 & 21 & 74 & 74.2 \\
\hline 20 & 234 & 73.0 & 21 & 3 & 73.9 & 21 & 76 & 73.3 \\
\hline 20 & 248 & 72.9 & 21 & 4 & 73.9 & 21 & 78 & 72.8 \\
\hline 20 & 278 & 73.0 & 21 & 6 & 73.0 & 21 & 83 & 73.4 \\
\hline 20 & 279 & 73.0 & 21 & 10 & 72.6 & 21 & 85 & 73.8 \\
\hline 20 & 295 & 74.2 & 21 & 13 & 73.9 & 21 & 86 & 73.3 \\
\hline 20 & 299 & 72.6 & 21 & 15 & 74.4 & 21 & 87 & 73.4 \\
\hline 20 & 318 & 73.9 & 21 & 17 & 72.8 & 21 & 89 & 73.0 \\
\hline 20 & 319 & 72.1 & 21 & 19 & 74.1 & 21 & 90 & 73.8 \\
\hline 20 & 324 & 73.2 & 21 & 22 & 73.3 & 21 & 91 & 74.1 \\
\hline
\end{tabular}


ESTUDIOS DEMOGRÁFICOS Y URBANOS, VOL. 32, NÚM. 1 (94), 2017, pp. 97-129

\section{Cuadro A1}

(continúa)

\begin{tabular}{|c|c|c|c|c|c|c|c|c|}
\hline Ent. & Mun. & $e_{0}$ & Ent. & Mun. & $e_{0}$ & Ent. & Mun. & $e_{0}$ \\
\hline 21 & 93 & 73.2 & 21 & 164 & 73.8 & 22 & 6 & 74.4 \\
\hline 21 & 94 & 73.8 & 21 & 166 & 73.8 & 22 & 7 & 73.9 \\
\hline 21 & 99 & 73.3 & 21 & 167 & 73.0 & 22 & 8 & 73.9 \\
\hline 21 & 100 & 73.0 & 21 & 169 & 73.3 & 22 & 9 & 73.8 \\
\hline 21 & 104 & 73.9 & 21 & 170 & 73.8 & 22 & 10 & 73.4 \\
\hline 21 & 106 & 73.0 & 21 & 172 & 73.3 & 22 & 11 & 74.3 \\
\hline 21 & 107 & 73.0 & 21 & 174 & 74.1 & 22 & 12 & 74.3 \\
\hline 21 & 108 & 73.8 & 21 & 175 & 73.8 & 22 & 13 & 73.8 \\
\hline 21 & 109 & 73.0 & 21 & 177 & 73.0 & 22 & 14 & 74.3 \\
\hline 21 & 110 & 73.1 & 21 & 178 & 73.0 & 22 & 16 & 74.3 \\
\hline 21 & 111 & 73.0 & 21 & 179 & 73.8 & 22 & 17 & 74.2 \\
\hline 21 & 112 & 73.2 & 21 & 180 & 73.8 & 22 & 18 & 73.8 \\
\hline 21 & 114 & 74.3 & 21 & 183 & 72.9 & 23 & 1 & 74.5 \\
\hline 21 & 115 & 73.9 & 21 & 186 & 73.8 & 23 & 2 & 73.9 \\
\hline 21 & 116 & 72.9 & 21 & 188 & 73.3 & 23 & 4 & 74.2 \\
\hline 21 & 117 & 74.2 & 21 & 189 & 73.8 & 23 & 5 & 74.9 \\
\hline 21 & 118 & 73.9 & 21 & 191 & 73.9 & 23 & 6 & 73.8 \\
\hline 21 & 119 & 74.3 & 21 & 194 & 73.8 & 23 & 7 & 73.8 \\
\hline 21 & 122 & 73.8 & 21 & 195 & 73.0 & 23 & 8 & 76.4 \\
\hline 21 & 124 & 73.8 & 21 & 197 & 73.8 & 23 & 9 & 74.7 \\
\hline 21 & 129 & 73.8 & 21 & 199 & 72.8 & 24 & 1 & 73.4 \\
\hline 21 & 132 & 74.2 & 21 & 202 & 73.0 & 24 & 3 & 72.9 \\
\hline 21 & 134 & 73.8 & 21 & 205 & 73.9 & 24 & 5 & 74.2 \\
\hline 21 & 138 & 73.9 & 21 & 207 & 73.8 & 24 & 7 & 73.8 \\
\hline 21 & 140 & 74.2 & 21 & 208 & 73.8 & 24 & 8 & 74.2 \\
\hline 21 & 142 & 73.8 & 21 & 211 & 74.2 & 24 & 10 & 73.8 \\
\hline 21 & 143 & 73.8 & 21 & 212 & 73.2 & 24 & 11 & 73.8 \\
\hline 21 & 145 & 73.0 & 21 & 213 & 73.5 & 24 & 12 & 73.0 \\
\hline 21 & 149 & 73.9 & 21 & 214 & 73.8 & 24 & 13 & 74.1 \\
\hline 21 & 153 & 73.8 & 21 & 217 & 73.0 & 24 & 14 & 73.3 \\
\hline 21 & 154 & 73.9 & 22 & 1 & 73.2 & 24 & 15 & 73.8 \\
\hline 21 & 156 & 74.2 & 22 & 2 & 73.4 & 24 & 16 & 73.8 \\
\hline 21 & 157 & 74.1 & 22 & 3 & 73.9 & 24 & 17 & 73.3 \\
\hline 21 & 161 & 73.8 & 22 & 4 & 73.8 & 24 & 18 & 73.3 \\
\hline 21 & 163 & 73.8 & 22 & 5 & 73.9 & 24 & 20 & 74.2 \\
\hline
\end{tabular}


Paredes y Silva, ESTIMACIÓN DE LA ESPERANZA DE VIDA

\section{Cuadro A1}

\section{(continúa)}

\begin{tabular}{|c|c|c|c|c|c|c|c|c|}
\hline Ent. & Mun. & $e_{0}$ & Ent. & Mun. & $e_{0}$ & Ent. & Mun. & $e_{0}$ \\
\hline 24 & 21 & 73.8 & 25 & 6 & 74.3 & 27 & 3 & 73.8 \\
\hline 24 & 22 & 73.4 & 25 & 7 & 73.4 & 27 & 4 & 74.2 \\
\hline 24 & 23 & 74.0 & 25 & 8 & 73.8 & 27 & 5 & 73.8 \\
\hline 24 & 24 & 73.9 & 25 & 9 & 74.2 & 27 & 6 & 73.9 \\
\hline 24 & 25 & 73.8 & 25 & 10 & 73.8 & 27 & 7 & 74.1 \\
\hline 24 & 27 & 74.0 & 25 & 11 & 74.2 & 27 & 8 & 73.9 \\
\hline 24 & 28 & 74.3 & 25 & 12 & 74.4 & 27 & 9 & 73.8 \\
\hline 24 & 29 & 72.9 & 25 & 13 & 73.9 & 27 & 10 & 74.2 \\
\hline 24 & 32 & 73.8 & 25 & 14 & 73.8 & 27 & 11 & 73.8 \\
\hline 24 & 34 & 73.8 & 25 & 15 & 74.6 & 27 & 12 & 73.8 \\
\hline 24 & 35 & 74.4 & 25 & 16 & 73.9 & 27 & 13 & 74.3 \\
\hline 24 & 36 & 73.4 & 25 & 17 & 73.8 & 27 & 14 & 74.2 \\
\hline 24 & 37 & 73.3 & 25 & 18 & 74.2 & 27 & 15 & 73.8 \\
\hline 24 & 38 & 73.4 & 26 & 2 & 74.3 & 27 & 16 & 73.8 \\
\hline 24 & 39 & 73.4 & 26 & 3 & 73.9 & 27 & 17 & 73.8 \\
\hline 24 & 40 & 73.8 & 26 & 12 & 73.8 & 28 & 1 & 73.8 \\
\hline 24 & 41 & 73.3 & 26 & 17 & 74.3 & 28 & 2 & 73.8 \\
\hline 24 & 42 & 73.8 & 26 & 18 & 74.4 & 28 & 3 & 74.4 \\
\hline 24 & 43 & 73.9 & 26 & 19 & 74.6 & 28 & 7 & 74.2 \\
\hline 24 & 45 & 73.9 & 26 & 25 & 74.7 & 28 & 9 & 74.4 \\
\hline 24 & 46 & 73.8 & 26 & 26 & 73.8 & 28 & 12 & 73.8 \\
\hline 24 & 49 & 73.8 & 26 & 29 & 74.5 & 28 & 13 & 73.8 \\
\hline 24 & 50 & 73.8 & 26 & 30 & 74.3 & 28 & 15 & 75.2 \\
\hline 24 & 51 & 73.9 & 26 & 33 & 73.8 & 28 & 16 & 73.9 \\
\hline 24 & 52 & 74.1 & 26 & 35 & 74.1 & 28 & 17 & 73.9 \\
\hline 24 & 53 & 73.8 & 26 & 36 & 74.5 & 28 & 19 & 74.0 \\
\hline 24 & 54 & 73.3 & 26 & 41 & 74.3 & 28 & 21 & 74.2 \\
\hline 24 & 55 & 73.8 & 26 & 42 & 74.1 & 28 & 22 & 74.3 \\
\hline 24 & 57 & 73.3 & 26 & 43 & 74.5 & 28 & 24 & 74.9 \\
\hline 24 & 58 & 73.8 & 26 & 48 & 74.3 & 28 & 25 & 74.5 \\
\hline 25 & 1 & 74.4 & 26 & 58 & 74.7 & 28 & 27 & 74.3 \\
\hline 25 & 2 & 74.2 & 26 & 70 & 74.3 & 28 & 30 & 73.9 \\
\hline 25 & 3 & 73.0 & 26 & 71 & 74.2 & 28 & 32 & 74.4 \\
\hline 25 & 4 & 73.8 & 27 & 1 & 73.8 & 28 & 33 & 74.6 \\
\hline 25 & 5 & 73.8 & 27 & 2 & 73.9 & 28 & 35 & 74.1 \\
\hline
\end{tabular}


ESTUDIOS DEMOGRÁFICOS Y URBANOS, VOL. 32, NÚM. 1 (94), 2017, pp. 97-129

\section{Cuadro A1}

\section{(continúa)}

\begin{tabular}{|c|c|c|c|c|c|c|c|c|}
\hline Ent. & Mun. & $e_{0}$ & Ent. & Mun. & $e_{0}$ & Ent. & Mun. & $e_{0}$ \\
\hline 28 & 37 & 73.9 & 30 & 10 & 73.1 & 30 & 55 & 73.4 \\
\hline 28 & 38 & 74.7 & 30 & 11 & 73.8 & 30 & 57 & 73.0 \\
\hline 28 & 39 & 73.9 & 30 & 13 & 74.2 & 30 & 58 & 73.2 \\
\hline 28 & 40 & 74.2 & 30 & 14 & 73.8 & 30 & 59 & 73.8 \\
\hline 28 & 41 & 74.4 & 30 & 15 & 73.8 & 30 & 60 & 73.8 \\
\hline 28 & 43 & 74.2 & 30 & 16 & 74.1 & 30 & 61 & 73.1 \\
\hline 29 & 2 & 74.1 & 30 & 21 & 73.8 & 30 & 62 & 73.8 \\
\hline 29 & 4 & 73.8 & 30 & 22 & 73.2 & 30 & 63 & 73.3 \\
\hline 29 & 5 & 74.5 & 30 & 23 & 73.3 & 30 & 65 & 74.2 \\
\hline 29 & 6 & 74.1 & 30 & 24 & 73.1 & 30 & 66 & 73.3 \\
\hline 29 & 7 & 73.9 & 30 & 25 & 72.8 & 30 & 68 & 74.2 \\
\hline 29 & 10 & 74.2 & 30 & 26 & 74.5 & 30 & 69 & 73.9 \\
\hline 29 & 13 & 74.2 & 30 & 27 & 73.4 & 30 & 70 & 73.3 \\
\hline 29 & 14 & 73.8 & 30 & 28 & 75.1 & 30 & 71 & 73.8 \\
\hline 29 & 15 & 74.2 & 30 & 29 & 72.8 & 30 & 72 & 73.3 \\
\hline 29 & 18 & 74.2 & 30 & 30 & 74.2 & 30 & 73 & 73.3 \\
\hline 29 & 19 & 74.2 & 30 & 31 & 73.2 & 30 & 75 & 73.3 \\
\hline 29 & 21 & 74.2 & 30 & 32 & 73.8 & 30 & 77 & 73.8 \\
\hline 29 & 23 & 73.8 & 30 & 33 & 73.3 & 30 & 78 & 73.0 \\
\hline 29 & 24 & 74.2 & 30 & 34 & 74.2 & 30 & 80 & 73.2 \\
\hline 29 & 25 & 74.3 & 30 & 35 & 73.3 & 30 & 81 & 73.9 \\
\hline 29 & 28 & 74.2 & 30 & 38 & 74.2 & 30 & 83 & 73.0 \\
\hline 29 & 31 & 74.2 & 30 & 39 & 74.3 & 30 & 85 & 74.2 \\
\hline 29 & 33 & 74.1 & 30 & 40 & 74.2 & 30 & 86 & 73.0 \\
\hline 29 & 34 & 73.8 & 30 & 43 & 73.3 & 30 & 87 & 74.4 \\
\hline 29 & 36 & 74.6 & 30 & 44 & 74.4 & 30 & 89 & 73.8 \\
\hline 29 & 38 & 73.6 & 30 & 45 & 74.2 & 30 & 91 & 73.3 \\
\hline 29 & 39 & 74.2 & 30 & 46 & 73.3 & 30 & 92 & 73.8 \\
\hline 29 & 41 & 74.2 & 30 & 47 & 72.9 & 30 & 94 & 73.8 \\
\hline 29 & 43 & 74.5 & 30 & 48 & 74.2 & 30 & 95 & 73.3 \\
\hline 29 & 44 & 74.5 & 30 & 49 & 73.8 & 30 & 97 & 74.2 \\
\hline 30 & 3 & 73.8 & 30 & 50 & 73.0 & 30 & 99 & 73.8 \\
\hline 30 & 4 & 73.9 & 30 & 51 & 73.3 & 30 & 100 & 73.8 \\
\hline 30 & 6 & 73.2 & 30 & 53 & 73.8 & 30 & 101 & 73.9 \\
\hline 30 & 9 & 73.9 & 30 & 54 & 73.9 & 30 & 102 & 73.8 \\
\hline
\end{tabular}


Paredes y Silva, ESTIMACIÓN DE LA ESPERANZA DE VIDA

\section{Cuadro A1}

\section{(continúa)}

\begin{tabular}{|c|c|c|c|c|c|c|c|c|}
\hline Ent. & Mun. & $e_{0}$ & Ent. & Mun. & $e_{0}$ & Ent. & Mun. & $e_{0}$ \\
\hline 30 & 103 & 72.8 & 30 & 149 & 72.4 & 30 & 208 & 74.2 \\
\hline 30 & 104 & 72.8 & 30 & 150 & 73.8 & 30 & 209 & 72.9 \\
\hline 30 & 105 & 74.2 & 30 & 151 & 73.9 & 30 & 210 & 72.8 \\
\hline 30 & 108 & 74.1 & 30 & 152 & 73.9 & 30 & 211 & 73.8 \\
\hline 30 & 109 & 73.8 & 30 & 154 & 73.3 & 30 & 212 & 73.0 \\
\hline 30 & 111 & 73.8 & 30 & 155 & 73.3 & 31 & 2 & 73.8 \\
\hline 30 & 112 & 73.8 & 30 & 157 & 73.3 & 31 & 19 & 72.8 \\
\hline 30 & 114 & 73.9 & 30 & 158 & 73.3 & 31 & 33 & 73.3 \\
\hline 30 & 115 & 74.2 & 30 & 159 & 72.2 & 31 & 38 & 73.9 \\
\hline 30 & 116 & 73.8 & 30 & 160 & 73.8 & 31 & 40 & 73.8 \\
\hline 30 & 117 & 73.8 & 30 & 161 & 73.4 & 31 & 41 & 74.5 \\
\hline 30 & 118 & 74.6 & 30 & 164 & 73.8 & 31 & 48 & 73.8 \\
\hline 30 & 120 & 73.8 & 30 & 167 & 73.3 & 31 & 50 & 74.5 \\
\hline 30 & 121 & 73.4 & 30 & 168 & 72.8 & 31 & 52 & 73.8 \\
\hline 30 & 122 & 73.1 & 30 & 169 & 73.8 & 31 & 56 & 73.8 \\
\hline 30 & 123 & 73.8 & 30 & 172 & 73.3 & 31 & 58 & 73.8 \\
\hline 30 & 124 & 73.8 & 30 & 173 & 73.3 & 31 & 59 & 74.2 \\
\hline 30 & 125 & 73.8 & 30 & 174 & 73.8 & 31 & 76 & 73.8 \\
\hline 30 & 126 & 73.8 & 30 & 175 & 73.8 & 31 & 79 & 73.8 \\
\hline 30 & 127 & 72.4 & 30 & 178 & 73.9 & 31 & 85 & 73.2 \\
\hline 30 & 128 & 73.9 & 30 & 181 & 73.9 & 31 & 89 & 73.8 \\
\hline 30 & 129 & 73.8 & 30 & 183 & 73.8 & 31 & 93 & 73.8 \\
\hline 30 & 130 & 73.3 & 30 & 188 & 73.3 & 31 & 96 & 73.8 \\
\hline 30 & 131 & 74.5 & 30 & 189 & 74.1 & 31 & 101 & 74.2 \\
\hline 30 & 132 & 73.8 & 30 & 191 & 74.2 & 31 & 102 & 73.8 \\
\hline 30 & 133 & 74.2 & 30 & 192 & 73.8 & 32 & 5 & 74.3 \\
\hline 30 & 134 & 73.8 & 30 & 193 & 74.3 & 32 & 7 & 74.2 \\
\hline 30 & 135 & 73.9 & 30 & 196 & 73.8 & 32 & 9 & 73.9 \\
\hline 30 & 138 & 74.4 & 30 & 197 & 73.3 & 32 & 10 & 74.1 \\
\hline 30 & 141 & 73.8 & 30 & 201 & 73.0 & 32 & 14 & 73.9 \\
\hline 30 & 142 & 73.3 & 30 & 203 & 73.0 & 32 & 16 & 73.8 \\
\hline 30 & 143 & 73.8 & 30 & 204 & 74.2 & 32 & 17 & 74.4 \\
\hline 30 & 144 & 73.8 & 30 & 205 & 73.8 & 32 & 19 & 74.2 \\
\hline 30 & 147 & 72.6 & 30 & 206 & 74.5 & 32 & 20 & 74.2 \\
\hline 30 & 148 & 73.8 & 30 & 207 & 73.8 & 32 & 22 & 74.2 \\
\hline
\end{tabular}




\section{Cuadro A1}

(concluye)

\begin{tabular}{|c|c|c|c|c|c|c|c|c|}
\hline Ent. & Mun. & $e_{0}$ & Ent. & Mun. & $e_{0}$ & Ent. & Mun. & $e_{0}$ \\
\hline 32 & 23 & 74.3 & 32 & 38 & 73.8 & 32 & 48 & 74.2 \\
\hline 32 & 24 & 74.2 & 32 & 39 & 74.2 & 32 & 49 & 73.9 \\
\hline 32 & 26 & 73.8 & 32 & 40 & 73.8 & 32 & 51 & 73.8 \\
\hline 32 & 29 & 74.2 & 32 & 42 & 73.8 & 32 & 52 & 74.2 \\
\hline 32 & 31 & 74.1 & 32 & 44 & 73.9 & 32 & 54 & 73.8 \\
\hline 32 & 34 & 74.2 & 32 & 45 & 74.0 & 32 & 55 & 74.2 \\
\hline 32 & 36 & 74.2 & 32 & 46 & 74.2 & 32 & 56 & 74.2 \\
\hline
\end{tabular}

Nota: Véase la versión completa de este anexo en: $<$ https://drive.google.com/file/d/0B Ro9fefIJtYSjJ1TnRucFhZMFE/view?usp=sharing>.

Fuente: Elaboración propia con base en INEGI (2010), Estadísticas vitales de defunciones, Base de datos, e INEGI (2010), Censo de Población y Vivienda 2010, Tabulado del cuestionario básico.

\section{Bibliografía}

Bravo, Jorge y Joana Malta (2010), "Estimating life expectancy in small population areas", ponencia presentada en la Conference of European Statisticians, UN Statistical Commission and Economic Commission for Europe, Lisboa, 28 a 30 de abril.

Chiang, Chin (1968), "The life table and its construction", en Chin Chiang (aut.), Introduction to stochastic processes in biostatistics, Nueva York, Wiley, pp. 189-214.

Congdon, Peter (2014a), "Estimating life expectancies for US small areas: A regression framework", Journal of Geographical Systems, vol. 16, núm. 1, pp. 1-18. Disponible en: < http://link.springer.com/article/10.1007/s10109-013-0177-4>.

Congdon, Peter (2014b), "Estimating life expectancy in small areas, with an application to recent changes in life expectancy in US counties", en Jon Anson y Marc Luy (eds.), Mortality in an international perspective, Suiza, Springer (European Studies of Population, 18). Disponible en: <http://link.springer.com/ chapter/10.1007\%2F978-3-319-03029-6_2\#page-1>.

Conapo (2011), Índice de marginación por entidad federativa y municipio 2010, México. Consejo Nacional de Población. Disponible en: <http://www.conapo. gob.mx/en/CONAPO/Indices_de_Marginacion_2010_por_entidad_federativa y_municipio> (10 de enero de 2015).

Conapo (2012), Proyecciones de la población 2010-2050, Base de datos, México, Instituto Nacional de Estadística y Geografía. Disponible en: <http://www. conapo.gob.mx/es/CONAPO/Proyecciones_Datos>.

Conapo (2015), Indicadores demográficos básicos, México, Consejo Nacional de Po- 
blación. Disponible en: <http://www.conapo.gob.mx/es/CONAPO/Proyecciones Datos $>$ (10 de enero de 2015).

Davidian, Marie y David Giltinan (1995), "Nonlinear models for repeated measurement data", Monographs on Statistics and Applied Probability, núm. 62, Nueva York, Chapman \& Hall/CRC. Disponible en: <http://www4.stat.ncsu. edu/ davidian/nlmmreview.pdf $>$.

Eayres, Daniel y Edward Williams (2003), "Evaluation of methodologies for small area life expectancy estimation", Journal of Epidemiology and Community Health, vol. 58, pp. 243-249. Disponible en: <http://www.ncbi.nlm.nih.gov/ pubmed/14966240>.

Flores, Miguel, Benjamin Bradshaw y Nazrul Hoque (2013), "Regional differences in life expectancy at birth in Mexican municipalities, 1990-2000", MPRA Paper, núm. 55212, p. 9. Disponible en: <https://mpra.ub.uni-muenchen.de/55212/>.

Guerrero, Víctor M. y Eliud Silva (2013), "Graduación no paramétrica con suavidad y estructura impuestas por el analista: aplicaciones demográficas para México", Estudios Demográficos y Urbanos, vol. 28, núm. 2 (83), pp. 429-468. Disponible en: <http://estudiosdemograficosyurbanos.colmex.mx./index.php/edu/article/ view/1434/1427>.

Haupt, Arthur y Thomas Kane (2003), Population handbook: Guía rápida de población, 4ta edición, Washington, D.C., Population Reference Bureau. Disponible en: $<$ http://www.prb.org/pdf/PopHandbook_Sp.pdf $>$.

INEGI (2001), Indicadores sociodemográficos de México, México, Instituto Nacional de Estadística y Geografía. Disponible en: <http://www.inegi.org.mx/ prod_serv/contenidos/espanol/bvinegi/productos/integracion/sociodemografico/ indisociodem/2001/indi2001.pdf $>$.

INEGI (2010a), Estadísticas vitales de defunciones, Base de datos, México, Instituto Nacional de Estadística y Geografía.

INEGI (2010b), Censo de Población y Vivienda 2010, Tabulados del cuestionario básico, México, Instituto Nacional de Estadística y Geografía.

Mazur, Peter (1972), "Using regression models to estimate the expectation of life for the U.S.S.R.”, Journal of the Americas Statistical Association, vol. 67, núm. 337, pp. 31-36. Disponible en: <http://www.jstor.org/stable/2284698?seq=1\#page scan_tab_contents>.

Ortega, Antonio (1982), Tablas de mortalidad, Serie B, núm. 1008, San José, Costa Rica, Centro Latinoamericano de Demografía. Disponible en: $<$ http://repositorio .cepal.org/handle/11362/8718>.

Ortega, Antonio (1987), Tablas de mortalidad, Serie B, núm. 1004, San José, Costa Rica Centro Latinoamericano de Demografía. Disponible en: <http://archivo. cepal.org/pdfs/1997/S9700588.pdf>.

PNUD (2012), Índice de Desarrollo Humano de Hogares e Individuos 2010, México, Oficina de Investigación en Desarrollo Humano, Programa de las Naciones Unidas para el Desarrollo.

Sarma, Rajan y Labananda Choudhury (2014), “A new model for estimating district 
life expectancy at birth in India with special reference to Assam state", Canadian Studies in Population, vol. 41, núms. 1-2, pp. 180-191. Disponible en: $<$ https:// ejournals.library.ualberta.ca/index.php/csp/article/viewFile/22170/16466>.

Silcocks Paul, D. A. Jenner y R. Reza (2001), "Life expectancy as a summary of mortality in a population: Statistical considerations and suitability for use by health authorities", Journal of Epidemiology and Community Health, vol. 55, núm. 1, pp. 38-43. Disponible en: <http://www.ncbi.nlm.nih.gov/pmc/articles/ PMC1731769/>.

Silva, Eliud y Víctor M. Guerrero (2016), "Penalized least squares smoothing of two-dimensional mortality tables with imposed smoothness", Journal of Applied Statistics. Disponible en: <https://doi.org/10.1080/02664763.2016.1221905>.

Swanson, David (1989), “A state-based regression model for estimating substate life expectancy”, Demography, vol. 26, núm. 1, pp. 161-170. Disponible en: $<$ https:// www.jstor.org/stable/2061502?seq=1\#page_scan_tab_contents $>$.

Swanson, David, Mary McGehee y Hoque Nazrul (2009), "Socio-economic status and life expectancy in the United States, 1970-1990", Population Review, vol. 48, núm. 2, pp. 41-65. Disponible en: <http://escholarship.org/uc/ item/2gc0c1xw\#page-2>.

Swanson, David y Lucky Tedrow (2012), "Using cohort change ratios to estimate life expectancy in populations with negligible migration: A new approach", Canadian Studies in Population, vol. 39, núms. 1-2, pp. 83-90. Disponible en: $<$ http://www.canpopsoc.ca/CanPopSoc/assets/File/publications/journal/2012/ CSPv39n1-2p83.pdf $>$.

Toson, Barbara y Allan Baker (2005), "Life expectancy at birth: methodological options for small populations", National Statistics Methodological Series, núm. 33, Newport, Office for National Statistics.

\section{Acerca de los autores}

Israel Paredes Morales es actuario de la Facultad de Ciencias Actuariales de la Universidad Anáhuac México. Se ha enfocado principalmente en el ramo asegurador, con particular interés en el reaseguro; en su ejercicio profesional, en varias ocasiones ha sido acreedor de premios por su desempeño dentro de la compañía aseguradora para la que labora. Ha realizado distintos trabajos de investigación en temas demográficos.

José Eliud Silva Urrutia es profesor investigador de la Facultad de Ciencias Actuariales de la Universidad Anáhuac México. Es actuario por la Universidad Nacional Autónoma de México (UNAM) y maestro en Demografía por El Colegio de México, A.C. Tiene doctorado en Ingeniería Matemática con especialidad en Estadística por la Universidad Carlos III de Madrid, 
donde obtuvo la nota más alta que se otorga en examen de grado. Se ha dedicado a la docencia, investigación y consultoría en temas estadísticos, además de haber trabajado en el sector asegurador y en la rama de la evaluación educativa. Es árbitro en revistas especializadas nacionales e internacionales, tanto en estadística como en demografía. Ha sido merecedor de reconocimientos en temas de investigación en el ámbito demográfico y actuarial. Actualmente es miembro del Sistema Nacional de Investigadores del Conacyt, nivel I. Recientemente ha sido nombrado vocal de la mesa directiva de la Asociación Mexicana de Estadística.

Fecha de recepción: 7 de octubre de 2015.

Fecha de aceptación: 10 de junio de 2016. 
